Old Dominion University

ODU Digital Commons

Mathematics \& Statistics Theses \&

Dissertations

Mathematics \& Statistics

Winter 1987

\title{
On the Thermal Stresses Due to a Uniform Heat Flow Past a Circular Hole With a Radial Edge Crack
}

George F. Edmonds

Old Dominion University

Follow this and additional works at: https://digitalcommons.odu.edu/mathstat_etds

Part of the Mathematics Commons

\section{Recommended Citation}

Edmonds, George F.. "On the Thermal Stresses Due to a Uniform Heat Flow Past a Circular Hole With a Radial Edge Crack" (1987). Doctor of Philosophy (PhD), Dissertation, Mathematics \& Statistics, Old Dominion University, DOI: 10.25777/rtt7-yn11 https://digitalcommons.odu.edu/mathstat_etds/87

This Dissertation is brought to you for free and open access by the Mathematics \& Statistics at ODU Digital Commons. It has been accepted for inclusion in Mathematics \& Statistics Theses \& Dissertations by an authorized administrator of ODU Digital Commons. For more information, please contact digitalcommons@odu.edu. 


\title{
ON THE THERMAL STRESSES DUE TO \\ A UNIFORM HEAT FLOW PAST A CIRCULAR HOLE WITH A RADIAI EDGE CRACK
}

\author{
by
}

GEORGE F. EDMONDS

B.S. June 1951, Hampton Institute, Hampton, VA

M.S. August 1957, New York University, New York, NY

A Dissertation Submitted to the Faculty of Old Dominion University in Partial Fulfilment of the Requirements for the Degree of

\author{
DOCTOR OF PHILOSOPHY \\ in \\ COMPUTATIONAL AND APPLIED MATHEMATICS
}

December, 1987

Approved by,

I. Tweed Tirertar) C 


\author{
ABSTRACT \\ On The Thermal Stresses Due To \\ A Uniform Heat Flow Past a \\ Circular Hole with a Radial Edge Crack
}

\author{
George F. Edmonds \\ Old Dominion University, 1987 \\ Director: Dr. John Tweed
}

The problem solved in this dissertation is that of finding the stresses in an isotropic, linear, thermoelastic solid when a uniform heat flow is disturbed by the presence of an insulated circular hole with a radial edge crack. By superimposing a Mellin transform solution of the equations of thermoelasticity on a Michell series solution the author reduces the problem to a pair of singular integral equations which are then solved numerically. The stress intensity factors and crack formation energies, quantities of interest to workers in fracture mechanics, are then calculated. 
To John Tweed ior all his help and guidance and to my wife for her patience. 


\section{ACKNOWLEDGEMENT}

I wish to express my appreciation to Dr. John Heinbockel, Dr. John Swetits, Dr. Philip Wohl and Dr. Stanley Weinstein for their advice, assistance and encouragement during my doctoral work and research. Dr. Heinbockel's assistance with my computer programming is particularly appreciated. I also wish to thank Dr. Larry Wilson and Dr. Gordon Melrose for their helpful questions.

I am indebted to Dr. John Tweed, dissertation director, for suggesting this line of research and for his patience, kindness and guidance throughout.

Finally, many thanks to Ms Barbara Jeffrey for her patience and ad̉vice and for typing this dissertation. 


\section{TABLE OF CONTENTS}

LIST OF FIGURES $\ldots \ldots \ldots \ldots \ldots \ldots \ldots \ldots \ldots \ldots \ldots \ldots \ldots$

CHAPTER 1. INTRODUCTION .......................... 1

CHAPTER 2. SOLUTIONS OF THE EQUATIONS OF

THERMOELASTICITY $. . \ldots \ldots \ldots \ldots \ldots \ldots \ldots \ldots \ldots \ldots, \ldots$

2.1 General Results .................................... 4

2.2 Michell's Solution of the Equations

of Thermoelasticity ................................. 8

2.3 The Mellin Transform Solution of the

Equations of Thermoelasticity.........................13

CHAPTER 3. STATEMENT OF THE PROBLEM ............. 15

3.1 The Problem ..................................... 15

3.2 Resolution into Problem 1 and Problem 2 .............. 13

CHAPTER 4. THE SOLUTION OF PROBLEM $1 \ldots \ldots \ldots \ldots \ldots . \ldots 2 \overline{1}$

4.1 The Temperature Field ................................21

4.2 The Mechanical Field ...............................27

CHAPTER 5. THE SOLUTION OF PROBLEM $2 \ldots \ldots \ldots \ldots \ldots 41$

CHAPTER 6. NUMERICAL PROCEDURES.................44

6.1 The Stress Intensities and the Crack

Formation Energies ............................... 44

6.2 The Computation of the Energy Weight Functions............45

6.3 Numerical Results ................................. 46

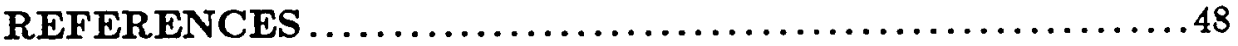

APPENDICES

A The Summmation of Series Involved in Calculating $f_{2}(r) \ldots 50$

B Some Useful Integrals .............................. 52

C The Program Stress ............................. 50

AUTOBIOGRAPHICAL STATEMENT ..................79 


\section{LIST OF FIGURES}

FIGURE

Page

1. Uniform heat flow past a circular hole with a radial edge crack ....16

2. The variation of $k_{1} / k_{0} \cos \theta_{0},-k_{2} / k_{0} \sin \theta_{0}, W_{1} / W_{0}$, and $W_{2} / W_{0}$ with relative crack length (C-B)/B. ............47 


\section{CHAPTER 1}

\section{INTRODUCTION}

The problem in this dissertation is that of finding the stress in an infnite, isotropic, homogeneous and linear thermoelastic solid when a unform heat flow

$$
g=q_{0}\left(\cos \theta_{0} \underset{i}{i}+\sin \theta_{0} j\right)
$$

is disturbed by the presence of an insulated circular hole $0 \leq R \leq B$, $0 \leq \theta \leq 2 \pi$ with a radial edge crack $B \leq R \leq C, \theta=0$.

The problem of determining the stresses near the tip of a crack at the edge of a circular hole in an infinite isothermal elastic solid appears to have been first investigated by Bowie [1]. Using an complex mapping technique Bowie obtained an approximate solution for the case of a pressurized crack. Thereafter, using Naylor's finite Miellin transform [3], Tweed [2] found an exact solution for the case in which the crack is subject to a longitudinal shear load. Tweed and Rooke [4] then showed that, in the plane strain case, the stress intensity factor and the crack formation energy are related to the solution of a weakly singular Fredholm equation. Using this technique, they calculated the stress intensity factors and crack formation energy to a high degree of accuracy.

Tweed and Rooke [5] have also solved the problem when the hole and the crack are subject to arbitrary in-plane loads. Via superposition, they have shown that this case is equivalent to that in which the hole is traction free and the crack is subject to loads of the form: 


$$
\begin{aligned}
& \sigma_{\theta \theta}(R, 0)=\sigma_{\theta \theta}(R, 2 \pi)=-p_{0} f_{1}\left(\frac{R}{B}\right), \quad B<R<C, \\
& \sigma_{R \theta}(R, 0)=\sigma_{R \theta}(R, 2 \pi)=-p_{0} f_{2}\left(\frac{R}{B}\right), \quad B<R<C .
\end{aligned}
$$

Here they reduce the problem to a pair of singular integral equations which are solved using numerical methods. A full discussion of the early work on crack problems of linear elasticity can be found in Sneddon and Lowengrub [6].

When a uniform heat flow in an infinite thermoelastic solid is disturbed by the presence of an insulated circular hole and radial edge crack, the resulting thermal stresses force the crack to open and may cause it to spread. The strategy for analysing this situation involves calculating the stresses at the proposed crack site when the crack is not piesent for, by linearity, these stresses may be interpreted as the crack opening loads in an equivalent isothermal elastic problem. Once these loads are determined the technique of Tweed and Rooke [5] can be used to calculate the stress intensity factors and the crack formation energy which are used to predict crack growth. Hence, a major portion of this dissertation is concerned with determining these loads.

In Chapter 2 a special case of Michell's solution as well as a Mellin transform solution of the equations of thermoelasticity are described. The special case of the Michell solution is due to Tweed who first derived it in reference [7].

In Chapter 3 the solution of the equations of thermoelasticity for the case in which a uniform heat flow is disturbed by an insulated circular hole is given. The author's problem then reduces to that of determining pertur- 
bations in this solution due to the presence of the crack. The problem of determining these perturbations is resolved into two component problems: PROBLEM 1.

In the absence of a crack and under the assumption that the hole and proposed crack site are thermally insulated determine the thermoelastic stresses occurring at the proposed site as a result of the disturbed heat flow.

\section{PROBLEM 2.}

Solve the equations of isothermal elasticity in the region external to the hole $0 \leq R \leq B, \quad 0 \leq \theta \leq 2 \pi$ and the crack $\theta=0, B \leq R<C$ when the crack is opened by the stress loadings obtained in Problem 1.

These problems are solved in Chapters 4 and 5, respectively. In Chapter 6 , the numerical procedures used are described and the numerical results are given.

There are three appendices. Appendix A contains sums of series used to calculate the shear load function $s_{r \theta}(r, 0)=f_{2}(r)$ on the crack. Appendix $B$ contains a table of integrals needed to evaluate the load functions and the crack formation energy. Appendix $\mathrm{C}$ contains the author's computer program STRESS, which was used to produce the numerical results described in Chapter 6.

The numbering of equations in the text is a standard one. Equations are numbered consecutively within a section by $[m . n]$ where $m$ is the section number, $n$ refers to the $n^{\text {th }}$ equation in the section. Equations are referenced by $(k . m . n)$ where $k$ is the number of the chapter in which the equation occurs. 


\section{CHAPTER 2}

\section{SOLUTIONS OF THE EQUATIONS OF THERMOELASTICITY}

\section{General Results}

We consider an isotropic, homogeneous thermoelastic solid which occupies the $(R, \theta)$ plane and is initially at temperature $T_{0}$ at which time it is free of stress and strain. If the solid is subjected to a uniform heat flow

$$
g=q_{0}\left\{\cos \theta_{0} \underset{\sim}{i}+\sin \theta_{0} i\right\}
$$

it then acquires a displacement field:

$$
\underset{\sim}{U}(R, \theta)=U_{R}(R, \theta) \underset{\sim}{\sim_{R}}+U_{\theta}(R, \theta) \underset{\sim}{\mathbb{\sim}_{\theta}}
$$

and a temperature field

$$
T=T_{0}+\Psi(R, \theta)
$$

which produce a strain field $e_{R R}(R, \theta), e_{R \theta}(R, \theta), e_{\theta \theta}(R, \theta)$ and a corresponding stress field $\sigma_{R R}(R, \theta), \sigma_{R \theta}(R, \theta), \sigma_{\theta \theta}(R, \theta)$.

As usual, we shall let $\kappa$ denote the thermal conductivity, $\alpha$ the coefficient of linear expansion, $\nu$ Poisson's ratio, $\mu$ the shear modulis, $E$ Young's modulus, and $\Phi(R, \theta)$ the Airy stress function. The strains are then related to the displacements by the equations:

$$
e_{R R}=\frac{\partial U_{R}}{\partial R}
$$




$$
\begin{gathered}
e_{R \theta}=\frac{1}{2}\left(\frac{1}{R} \frac{\partial U_{R}}{\partial \theta}+\frac{\partial U_{\theta}}{\partial R}-\frac{U_{\theta}}{R}\right) \\
e_{\theta \theta}=\frac{1}{R} \frac{\partial U_{\theta}}{\partial \theta}+\frac{U_{R}}{R}
\end{gathered}
$$

and the stress-strain relations are given by

$$
\begin{gathered}
e_{R R}=\frac{1+\nu}{E}\left[(1-\nu) \sigma_{\theta \theta}-\nu \sigma_{R R}\right]+(1+\nu) \alpha \Psi \\
e_{R \theta}=\frac{1+\nu}{E} \sigma_{R \theta} \\
e_{\theta \theta}=\frac{1+\nu}{E}\left[(1-\nu) \sigma_{\theta \theta}-\nu \sigma_{R R}\right]+(1+\nu) \alpha \Psi .
\end{gathered}
$$

We can show [10] that, in the absence of body forces, the equations of equilibrium:

$$
\begin{gathered}
\frac{\partial \sigma_{R R}}{\partial R}+\frac{1}{R} \frac{\partial \sigma_{R \theta}}{\partial \theta}+\frac{\sigma_{R R}-\sigma_{\theta \theta}}{R}=0 \\
\frac{\partial \sigma_{R \theta}}{\partial R}+\frac{1}{R} \frac{\partial \sigma_{\theta \theta}}{\partial \theta}+\frac{2}{R} \sigma_{R \theta}=0
\end{gathered}
$$

are satisfied if and only if the stresses are derived from an Airy stress function $\Phi(R, \theta)$ in the following manner: 


$$
\begin{gathered}
\sigma_{R R}=\frac{1}{R} \frac{\partial \Phi}{\partial R}+\frac{1}{R^{2}} \frac{\partial^{2} \Phi}{\partial \theta^{2}} \\
\sigma_{R \theta}=-\frac{\partial}{\partial R}\left(\frac{1}{R} \frac{\partial \Phi}{\partial \theta}\right) \\
\sigma_{\theta \theta}=\frac{\partial^{2} \Phi}{\partial R^{2}}
\end{gathered}
$$

where $\Phi(R, \theta)$ satisfies the biharmonic equation

$$
\nabla^{4} \Phi(R, \theta)=0
$$

while the temperature field must satisfy Laplace's equation

$$
\nabla^{2} \Psi(R, \theta)=0
$$

and, for our problem, be periodic with period $2 \pi$ in $\theta$.

Before going on to determine the temperature field and the stress field we find it convenient to introduce the dimensionless quantities:

$$
\begin{aligned}
r=\frac{R}{B}, c=\frac{C}{B}, & \Theta(r, \theta)=\frac{1}{T_{0}} \Psi(R, \theta) \\
u(r, \theta)=\frac{E U_{R}(R, \theta)}{(1+\nu) p_{0} B}, & v(r, \theta)=\frac{E U_{\theta}(R, \theta)}{(1+\nu) p_{0} B} \\
s_{r r}(r, \theta)=\frac{\sigma_{R R}(R, \theta)}{p_{0}}, & s_{r \theta}(r, \theta)=\frac{\sigma_{R \theta}(R, \theta)}{p_{0}} \\
s_{\theta \theta}(r, \theta)=\frac{\sigma_{\theta \theta}(R, \theta)}{p_{0}}, & \phi(r, \theta)=\frac{\Phi(R, \theta)}{p_{0} B^{2}}
\end{aligned}
$$


in which $B$ is a characteristic length, and $p_{0}$ is a constant having dimensions of stress. In our problem $B$ is taken to be the radius of the hole while $p_{0}$ is given by

$$
p_{0}=\frac{E \alpha B q_{0}}{2 \kappa(1-\nu)}
$$




\section{Michell's Solution of the Equations of Thermoelasticity}

By using separation of variables it is not difficult to show [7] that the temperature is given by

$$
\begin{aligned}
\Theta(r, \theta)=A_{0} & +B_{0} \ln r+\sum_{n=1}^{\infty}\left(A_{n} r^{n}+B_{n} r^{-n}\right) \cos n \theta \\
& +\sum_{n=1}^{\infty}\left(A_{n}^{\prime} r^{n}+B_{n}^{\prime} r^{-n}\right) \sin n \theta
\end{aligned}
$$

the Airy stress function by

$$
\begin{aligned}
\phi(r, \theta) & =\left\{a_{0}+b_{0} \ln r+c_{0} r^{2}+d_{0} r^{2} \ln r\right\} \\
& +\left\{a_{0}^{\prime}+b_{0}^{\prime} \ln r+c_{0}^{\prime} r^{2}+d_{0}^{\prime} r^{2} \ln r\right\} \theta \\
& +\left\{a_{1} r+b_{1} r^{-1}+c_{1} r^{3}+d_{1} r \ln r\right\}\left\{\begin{array}{c}
\cos \theta \\
\sin \theta
\end{array}\right\} \\
& +\left\{a_{1}^{\prime} r+d_{1}^{\prime} r \ln r\right\}\left\{\begin{array}{l}
\theta \cos \theta \\
\theta \sin \theta
\end{array}\right\} \\
& +\sum_{n=2}^{\infty}\left\{a_{n} r^{n}+b_{n} r^{-n}+c_{n} r^{2+n}+d_{n} r^{2-n}\right\}\left\{\begin{array}{l}
\cos n \theta \\
\sin n \theta
\end{array}\right\},
\end{aligned}
$$

and the corresponding stresses by 


$$
\begin{aligned}
& s_{r r}(r, \theta)=\frac{1}{r} \frac{\partial \phi}{\partial r}+\frac{1}{r^{2}} \frac{\partial^{2} \phi}{\partial \theta^{2}} \\
& =\left\{b_{0} r^{-2}+2 c_{0}+d_{0}[1+2 \ln r]\right\} \\
& +\left\{b_{0}^{\prime} r^{-2}+2 c_{0}^{\prime}+d_{0}^{\prime}[1+2 \ln r]\right\} \theta \\
& +\left\{-2 b_{1} r^{-3}+2 c_{1} r+d_{1} r^{-1}\right\}\left\{\begin{array}{c}
\cos \theta \\
\sin \theta
\end{array}\right\} \\
& +\left\{2 a_{1}^{\prime} \tau^{-1}+2 d_{1}^{\prime} \tau^{-1} \ln r\right\}\left\{\begin{array}{r}
-\sin \theta \\
\cos \theta
\end{array}\right\} \\
& +d_{1}^{\prime} r^{-1}\left\{\begin{array}{c}
\theta \cos \theta \\
\theta \sin \theta
\end{array}\right\}-\sum_{n=2}^{\infty}\left\{n(n-1) a_{n} r^{n-2}\right. \\
& +n(n+1) b_{n} r^{-n-2}+(n+1)(n-2) c_{n} r^{n} \\
& \left.+(n-1)(n+2) d_{n} r^{-n}\right\}\left\{\begin{array}{l}
\cos n \theta \\
\sin n \theta
\end{array}\right\} \\
& s_{r \theta}(r, \theta)=-\frac{\partial}{\partial r}\left(\frac{1}{r} \frac{\partial \phi}{\partial \theta}\right) \\
& =\left\{a_{0}^{\prime} r^{-2}-b_{0}^{\prime} r^{-2}[1-\ln r]-c_{0}^{\prime}-d_{0}^{\prime}[1+\ln r]\right\} \\
& +\left\{2 b_{1} r^{-3}-2 c_{1} r-d_{1} r^{-1}\right\}\left\{\begin{array}{c}
-\sin \theta \\
\cos \theta
\end{array}\right\}-d_{1}^{\prime} r^{-1}\left\{\begin{array}{c}
\cos \theta-\theta \sin \theta \\
\sin \theta+\theta \cos \theta
\end{array}\right\} \\
& +\sum_{n=2}^{\infty}\left\{n(n-1) a_{n} r^{n-2}-n(n+1) b_{n} r^{-n-2}\right. \\
& \left.+n(n+1) c_{n} r^{n}-n(n-1) d_{n} r^{-n}\right\}\left\{\begin{array}{c}
\sin n \theta \\
-\cos n \theta
\end{array}\right\},
\end{aligned}
$$

9 


$$
\begin{aligned}
s_{\theta \theta}(r, \theta) & =\frac{\partial^{2} \phi}{\partial r^{2}} \\
& =\left\{-b_{0} r^{-2}+2 c_{0}+d_{0}[2 \ln r+3]\right\} \\
& +\left\{-b_{0}^{\prime} r^{-2}+2 c_{0}^{\prime}+d_{0}^{\prime}[2 \ln r+3]\right\} \theta \\
& +\left\{2 b_{1} r^{-3}+6 c_{1} r+d_{1} r^{-1}\right\}\left\{\begin{array}{cc}
\cos \theta \\
\sin \theta
\end{array}\right\}+d_{1}^{\prime} r^{-1}\left\{\begin{array}{c}
\theta \cos \theta \\
\theta \sin \theta
\end{array}\right\} \\
& +\sum_{n=2}^{\infty}\left\{n(n-1) a_{n} r^{n-2}+n(n+1) b_{n} r^{-n-2}\right. \\
& \left.+(n+2)(n+1) c_{n} r^{n}+(n-1)(n-2) d_{n} r^{-n}\right\}\left\{\begin{array}{c}
\cos n \theta \\
\sin n \theta
\end{array}\right\} .
\end{aligned}
$$

We note that the stresses are single-valued if and only if $b_{0}^{\prime}=c_{0}^{\prime}=d_{0}^{\prime}=$ $d_{1}^{\prime}=0$.

In order to determine the displacement field it is necessary to integrate the stress displacement relations

$$
\begin{gathered}
\frac{\partial u}{\partial r}=(1-\nu) s_{r r}-\nu s_{\theta \theta}+\frac{E \alpha T_{0}}{p_{0}} \Theta(r, \theta), \\
\frac{\partial v}{\partial \theta}=\left[(1-\nu) s_{\theta \theta}-\nu s_{r r}\right] r+\frac{E \alpha T_{0}}{p_{0}} r \Theta(r, \theta)-u, \\
s_{r \theta}=\frac{1}{2}\left[\frac{1}{r} \frac{\partial u}{\partial \theta}+r \frac{\partial}{\partial r}\left(\frac{v}{r}\right)\right]
\end{gathered}
$$

which are obtained by substituting from equations [1.4-1.6] into [1.7-1.9! After some effort this yields the following displacements corresponding to single-valued stresses to within an aribtrary translation or rotation: 


$$
\begin{aligned}
& u(r, \theta)=\left\{-b_{0} r^{-1}+2(1-2 \nu) c_{0} r+d_{0} r[2(1-2 \nu) \ln r-1]\right\} \\
& +\frac{E \alpha T_{0}}{p_{0}} r\left\{A_{0}+B_{0}[\ln r-1]\right\} \\
& +\left\{b_{1} r^{-2}+c_{1}(1-4 \nu) r^{2}+d_{1}[(1-2 \nu) \operatorname{in} r-2(1-\nu)]\right\}\left\{\begin{array}{l}
\cos \theta \\
\sin \theta
\end{array}\right\} \\
& +2(1-\nu) d_{1}\left\{\begin{array}{r}
\theta \sin \theta \\
-\theta \cos \theta
\end{array}\right\}+a_{1}^{\prime}[2(1-\nu) \ln r-(1-2 \nu)]\left\{\begin{array}{r}
-\sin \theta \\
\cos \theta
\end{array}\right\} \\
& +(1-2 \nu) a_{1}^{\prime}\left\{\begin{array}{l}
\theta \cos \theta \\
\theta \sin \theta
\end{array}\right\}+\frac{E \alpha T_{0}}{p_{0}}\left\{\frac{1}{2} A_{1} r^{2}+B_{1}[\ln r-1]\right\}\left\{\begin{array}{l}
\cos \theta \\
\sin \theta
\end{array}\right\} \\
& +\frac{E \alpha T_{0}}{p_{0}} B_{1}\left\{\begin{array}{r}
\theta \sin \theta \\
-\theta \cos \theta
\end{array}\right\} \\
& +\sum_{n=2}^{\infty}\left[\left\{-n a_{n} r^{n-1}+n b_{n} r^{-n-1}-c_{n}[n-2+4 \nu] r^{n+1}\right.\right. \\
& \left.\left.+d_{n}[n+2-4 \nu] r^{-n+1}\right\}\right]\left\{\begin{array}{c}
\cos n \theta \\
\sin n \theta
\end{array}\right\} \\
& +\frac{E \alpha T_{0}}{p_{0}}\left\{\frac{A_{n}}{n+1} r^{n+1}+\frac{B_{n}}{1-n} r^{1-n}\right\}\left\{\begin{array}{c}
\cos n \theta \\
\sin n \theta
\end{array}\right\}
\end{aligned}
$$




$$
\begin{aligned}
v(r, \theta) & =\left\{4(1-\nu) d_{0} r \theta-a_{0}^{\prime} r^{-1}+4(1-\nu) c_{0}^{\prime} r \ln r\right\}+\frac{E \alpha T_{0}}{p_{0}} B_{0} r \theta \\
& +\left\{b_{1} r^{-2}+(5-4 \nu) c_{1} r^{2}+(1-2 \nu) d_{1}[1-\ln r]\right\}\left\{\begin{array}{c}
\sin \theta \\
-\cos \theta
\end{array}\right\} \\
& +2(1-\nu) d_{1}\left\{\begin{array}{l}
\theta \cos \theta \\
\theta \sin \theta
\end{array}\right\}-2 a_{1}^{\prime}[\nu+(1-\nu) \ln r]\left\{\begin{array}{cc}
\cos \theta \\
\sin \theta
\end{array}\right\} \\
& -(1-2 \nu) a_{1}^{\prime}\left\{\begin{array}{c}
\theta \sin \theta \\
-\theta \cos \theta
\end{array}\right\}+\frac{E \alpha T_{0} B_{1}}{p_{0}}\left\{\begin{array}{l}
\cos \theta \\
\theta \sin \theta
\end{array}\right\} \\
& +\frac{E \alpha T_{0}}{p_{0}}\left\{\frac{1}{2} A_{1} r^{2}+B_{1}[1-\ln r]\right\}\left\{\begin{array}{c}
\sin \theta \\
-\cos \theta
\end{array}\right\} \\
& -\sum_{n=2}^{\infty}\left\{n a_{n} r^{n-1}+n b_{n} r^{-n-1}+[n+4-4 \nu] c_{n} r^{n+1}\right. \\
& \left.+[n-4+4 \nu] d_{n} r^{-n+1}\right\}\left\{\begin{array}{c}
-\sin n \theta \\
\cos n \theta
\end{array}\right\} \\
& +\frac{E \alpha T_{0}}{p_{0}}\left\{\frac{A_{n}}{n+1} r^{n+1}-\frac{B_{n}}{1-n} r^{1-n}\right\}\left\{\begin{array}{c}
\sin n \theta \\
-\cos n \theta
\end{array}\right\} .
\end{aligned}
$$




\section{The Mellin Transform Solution of the Equations of Thermoelasticity}

We introduce the Mellin transform pair [9]

$$
\begin{aligned}
\bar{\phi}(s, \theta) & =M[\phi(r, \theta) ; \quad r \rightarrow s] \\
& =\int_{0}^{\infty} \phi(r, \theta) r^{s-1} d r
\end{aligned}
$$

and

$$
\begin{aligned}
\phi(r, \theta) & =M^{-1}[\bar{\phi}(s, \theta) ; s \rightarrow r] \\
& =\frac{1}{2 \pi i} \int_{c-i \infty}^{c+i \infty} \bar{\phi}(s, \theta) r^{-s} d s .
\end{aligned}
$$

In terms of this transformation it can be shown [10] that, with an appropriate choice for $c=\operatorname{Re}(s)$, the temperature field for the problem described in section 2.1 is given by

$$
\overline{r^{2} \Theta}(s, \theta)=N(s) \cos (s+2) \theta+L(s) \sin (s+2) \theta
$$

and the Airy stress function by

$$
\begin{aligned}
\bar{\phi}(s, \theta) & =A(s) \sin s \theta+B(s) \cos s \theta \\
& +C(s) \sin (s+2) \theta+D(s) \cos (s+2) \theta
\end{aligned}
$$

where $A(s), B(s), C(s), D(s), N(s)$, and $L(s)$ are arbitrary functions of the parameter $s$ but are independent of $\theta$. The corresponding stresses and displacements are given by 


$$
\begin{aligned}
& s_{r r}(r, \theta)=r^{-2} M^{-1}\left[\frac{\partial^{2} \bar{\phi}(s, \theta)}{\partial \theta^{2}}-s \bar{\phi}(s, \theta) ; s \rightarrow r\right] \\
& s_{r \theta}(r, \theta)=r^{-2} M^{-1}\left[(s+1) \frac{\partial \bar{\phi}(s, \theta)}{\partial \theta} ; s \rightarrow r\right] \\
& s_{\theta \theta}(r, \theta)=r^{-2} M^{-1}[s(s+1) \bar{\phi}(s, \theta) ; s \rightarrow r] \\
& u(r, \theta)=-r^{-1} M^{-1}\left[( s + 1 ) ^ { - 1 } \left\{(1-\nu) \frac{\partial^{2} \bar{\phi}(s, \theta)}{\partial \theta^{2}}-s(1+\nu s) \bar{\phi}(s, \theta)\right.\right. \\
& \left.\left.+\frac{E \alpha T_{0}}{p_{0}} \bar{\theta}(s+2, \theta)\right\} ; s \rightarrow r\right]
\end{aligned}
$$

and

$$
\begin{aligned}
v(r, \theta) & =-r^{-1} M^{-1}\left[( s + 1 ) ^ { - 1 } ( s + 2 ) ^ { - 2 } \left\{(1-\nu) \frac{\partial^{3} \bar{\phi}(s, \theta)}{\partial \theta^{3}}\right.\right. \\
& +\left[(1-\nu) s^{2}+(s+1)(s+2)\right] \frac{\partial \bar{\phi}(s, \theta)}{\partial \theta} \\
& \left.\left.+\frac{E \alpha T_{0}}{p_{0}} \frac{\partial \bar{\Theta}(s+2, \theta)}{\partial \theta}\right\} ; s \rightarrow r\right] .
\end{aligned}
$$




\section{CHAPTER 3}

\section{STATEMENT OF THE PROBLEM}

\section{The Problem}

Consider a two-dimensional, linear, isotropic, thermoelastic solid which occupies the $(r, \theta)$ plane and contains a circular hole $0 \leq r \leq 1,0 \leq \theta \leq 2 \pi$ with a radial edge crack $1 \leq r \leq c, \theta=0$. We assume that the solid is initally unstressed and has uniform constant temperature $T_{0}$. Under conditions of plane strain we shall determine the thermal stresses in the vicinity of the crack tip when a uniform heat flow

$$
g=q_{0}\left(\cos \theta_{0} \underset{\sim}{i}+\sin \theta_{0} \underset{j}{j}\right)
$$

is disturbed by the crack and the hole which are thermally insulated [Fig. 1]. As in Chapter 2, we denote the temperature at the point $(r, \theta)$ by the symbol

$$
T(r, \theta)=T_{0}[1+\Theta(r, \theta)]
$$

Parkus [8] has shown that, for flow past an insulated circular hole, the thermoelastic field is given by

$$
\begin{aligned}
& \Theta^{0}(r, \theta)=-\frac{q_{0} B}{\kappa T_{0}}\left(r+r^{-1}\right) \cos \left(\theta-\theta_{0}\right), \\
& \phi^{0}(r, \theta)=\left(\frac{1}{2} r^{-1}+r \ln r\right) \cos \left(\theta-\theta_{0}\right),
\end{aligned}
$$




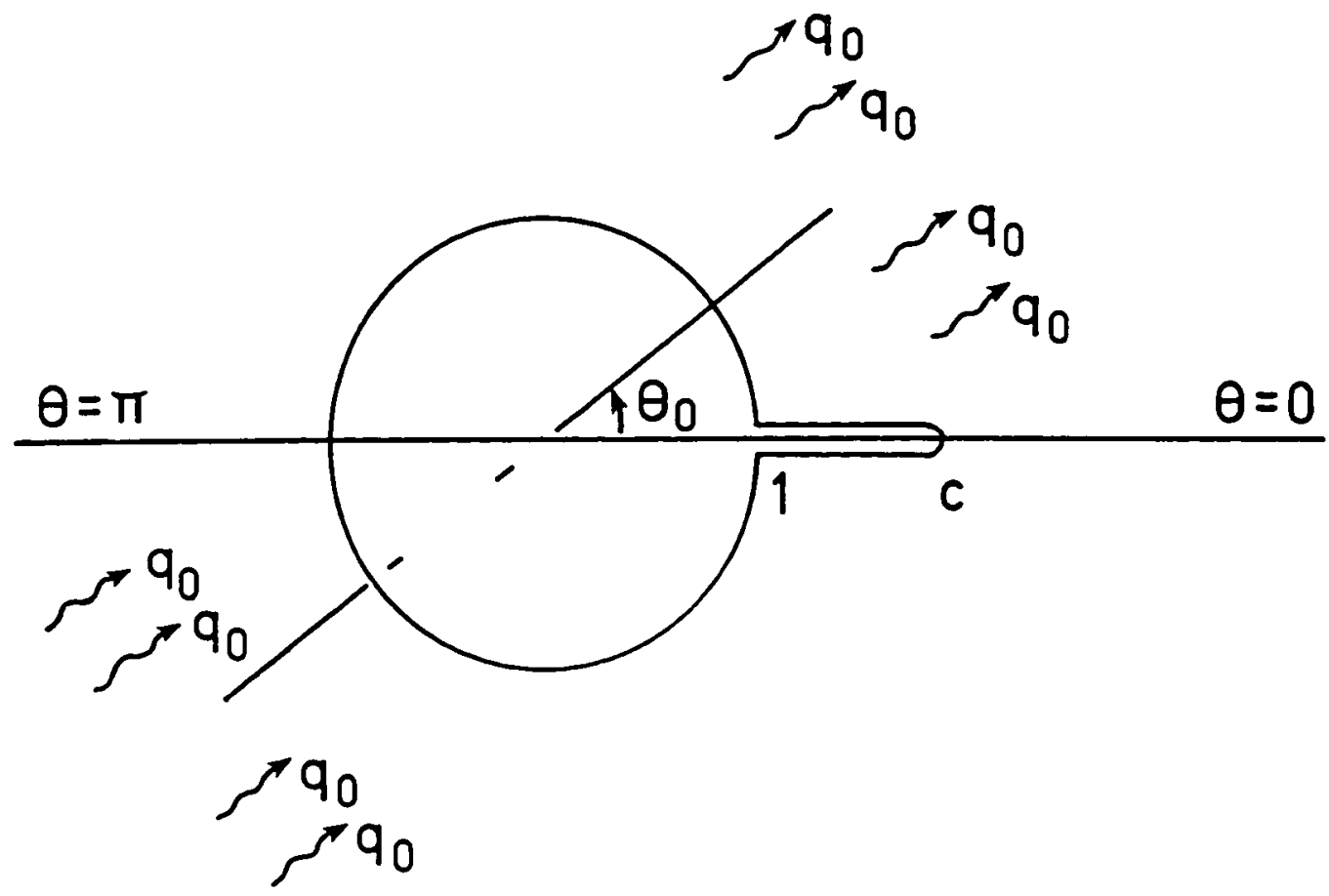

Figure 1. Uniform heat flow past a circular hole with a radial edge crack. 


$$
\begin{aligned}
u^{0}(r, \theta)= & \frac{1}{2}\left\{r^{-2}+(1-2 \nu) \ln r-4(1-\nu)\right\} \cos \left(\theta-\theta_{0}\right) \\
- & (1-\nu)\left\{r^{2}+2 \ln r-2\right\} \cos \left(\theta-\theta_{0}\right), \\
v^{0}(r, \theta)= & \frac{1}{2}\left\{r^{-2}+(1-2 \nu)[2-2 \ln r]\right\} \sin \left(\theta-\theta_{0}\right) \\
& -(1-\nu)\left\{r^{2}+2-2 \ln r\right\} \sin \left(\theta-\theta_{0}\right), \\
& s_{r r}^{0}(r, \theta)=\left(r^{-1}-r^{-3}\right) \cos \left(\theta-\theta_{0}\right), \\
& s_{r \theta}^{0}(r, \theta)=\left(r^{-1}-r^{-3}\right) \sin \left(\theta-\theta_{0}\right),
\end{aligned}
$$

and

$$
s_{\theta \theta}^{0}=\left(r^{-1}-r^{-3}\right) \cos \left(\theta-\theta_{0}\right) \text {. }
$$

In the presence of the crack the above solution is perturbed. Hence, our problem may be stated as follows: 


\section{PROBLEM}

Solve the dimensionless, plane-strain equations of linear thermoelasticity in the region $r>1,0<\theta<2 \pi$ subject to the boundary conditions:

1. At infinity $\Theta(r, \theta)=\Theta^{0}(r, \theta)+O\left(r^{-1}\right)$ and $\phi(r, \theta)=\phi^{0}(r, \theta)+O(r \ln r)$

2. $s_{r r}(1, \theta)=s_{r \theta}(1, \theta)=0$,

$$
0<\theta<2 \pi
$$

3. $s_{\theta \theta}(r, 2 \pi)-s_{\theta \theta}(r, 0)=0$,

$$
r>1
$$

$s_{r \theta}(r, 2 \pi)-s_{r \theta}(r, 0)=0$,

$$
r>1
$$

4. $s_{\theta \theta}(r, 0)=s_{r \theta}(r, 0)=0$,

$1<r<c$

$u(r, 2 \pi)-u(r, 0)=0$,

$c<r<\infty$

$v(r, 2 \pi)-v(r, 0)=0$,

$c<r<\infty$

$\lim _{r \rightarrow 1^{+}}\left[\frac{\partial u(r, 2 \pi)}{\partial r}-\frac{\partial u(r, 0)}{\partial r}\right] \quad<\infty$

$\lim _{r \rightarrow 1^{+}}\left[\frac{\partial v(v, 2 \pi)}{\partial r}-\frac{\partial v(r, 0)}{\partial r}\right] \quad<\infty$

5. $\frac{\partial \Theta(1, \theta)}{\partial r}=0$

$0<\theta<2 \pi$

6. $\frac{\partial \Theta(r, 2 \pi)}{\partial \theta}-\frac{\partial \Theta(r, 0)}{\partial \theta}=0, \quad \quad r>1$,

7. $\frac{\partial \Theta}{\partial \theta}(r, 0)=0, \quad 1<r<c$

$$
\Theta(r, 2 \pi)-\Theta(r, 0)=0, \quad c<r<\infty
$$




\section{Resolution into Problem 1 and Problem 2}

Using linearity,the solution of this problem is obtained by superimposing the solutions to problems 1 and 2 below.

\section{PROBLEM 1}

Solve the dimensionless, plane-strain equations of linear thermoelasticity in the region $r>1,0<\theta<2 \pi$ subject to the boundary conditions:

1. At infinity $\Theta(r, \theta)=\Theta^{0}(r, \theta)+O\left(r^{-1}\right)$ and $\phi(r, \theta)=\phi^{0}(r, \theta)+0(r \ln r)$,

2. $s_{r r}(1, \theta)=s_{r \theta}(1, \theta)=0$, $0<\theta<2 \pi$

3. $s_{\theta \theta}(r, 2 \pi)-s_{\theta \theta}(r, 0)=0$, $r>1$, $s_{r \theta}(r, 2 \pi)-s_{r \theta}(r, 0)=0$

$$
r>1,
$$

4. $u(r, 2 \pi)-u(r, 0)=0$,

$r>1$, $v(r, 2 \pi)-v(r, 0)=0$,

$r>1$,

5. $\frac{\partial \Theta(1, \theta)}{\partial r}=0$ $0<\theta<2 \pi$

6. $\frac{\partial \Theta(r, 2 \pi)}{\partial \theta}-\frac{\partial \Theta(r, 0)}{\partial \theta}=0, \quad \quad r>1$

7. $\frac{\partial \Theta(r, 0)}{\partial \theta}=0$ $1<r<c$ $\Theta(r, 2 \pi)-\Theta(r, 0)=0$ $c<r<\infty$. 
Calculate: $s_{\theta \theta}(r, 0)=f_{1}(r) \cos \theta_{0}$ and

$$
s_{r \theta}(r, 0)=f_{2}(r) \sin \theta_{0} \text { for } \quad 1<r<c .
$$

\section{PROBLEM 2}

Solve the dimensionless, plane-strain equations of linear isothermal elasticity in the region $r>1,0<\theta<2 \pi$ subject to the boundary conditions:

1. At infinity $\phi(r, \theta)=0(\ln r)$,

2. $s_{r r}(1, \theta)=s_{r \theta}(1, \theta)=0, \quad 0<\theta<2 \pi$,

3. $s_{\theta \theta}(r, 2 \pi)-s_{\theta \theta}(r, 0)=0, \quad r>1$,

$$
s_{r \theta}(r, 2 \pi)-s_{r \theta}(r, 0)=0, \quad r>1,
$$

4. $s_{\theta \theta}(r, 0)=-f_{1}(r) \cos \theta_{0}, \quad 1<r<c$,

$$
\begin{array}{ll}
s_{r \theta}(r, 0)=-f_{2}(r) \sin \theta_{0}, & 1<r<c, \\
u(r, 2 \pi)-u(r, 0)=0, & c<r<\infty, \\
v(r, 2 \pi)-v(r, 0)=0, & c<r<\infty, \\
\lim _{r \rightarrow 1^{+}}\left[\frac{\partial u(r, 2 \pi)}{\partial r}-\frac{\partial u(r, 0)}{\partial r}\right] & <\infty, \\
\lim _{r \rightarrow 1^{+}}\left[\frac{\partial v(r, 2 \pi)}{\partial r}-\frac{\partial v(r, 0)}{\partial r}\right] & <\infty .
\end{array}
$$




\section{CHAPTER 4}

\section{The Solution of Problem 1}

\section{The Temperature Field}

We solve problem 1 by perturbing the solution [3.1.2] - [3.1.8] for flow past an insulated circular hole. By inspection we see that the horizontal component of this solution already satisfies the conditions of problem 1 and hence only the vertical component need be perturbed. In addition, by symmetry, we find that the perturbations in the temperature and stress function must be anti-symmetric with respect to the rays $\theta=0$ and $\theta=\pi$. We begin by examining the temperature field which is assumed to be of the form:

$$
\Theta(r, \theta)=-\frac{B q_{0}}{\kappa T_{0}}\left(r+r^{-1}\right) \cos \left(\theta-\theta_{0}\right)+\Theta^{1}(r, \theta)
$$

It is clear that this field satisfies conditions $1,5,6$, and 7 if $\Theta^{1}(r, \theta)$ is given by the mixed boundary value problem:

$$
\begin{aligned}
& \nabla^{2} \Theta^{1}(r, \theta)=0, \quad r>1, \quad 0<\theta<\pi \\
& \text { i. } \Theta^{1}(r, \theta)=0\left(r^{-1}\right) \quad \text { at infinity, } \\
& \text { ii. } \quad \frac{\partial \Theta^{1}(1, \theta)}{\partial r}=0, \quad 0<\theta<\pi, \\
& \text { iii. } \Theta^{1}(r, \pi)=0, \quad r>1, \\
& \text { iv. } \quad \frac{\partial \Theta^{1}(r, 0)}{\partial \theta}=\frac{B q_{0} \sin \theta_{0}}{\kappa T_{0}}\left(r+r^{-1}\right), 1<r<c, \\
& \Theta^{1}(r, 0)=0, \quad r>c .
\end{aligned}
$$


The boundary value problem (1.2) is solved by making use of the Naylor transform [3] defined as follows:

$$
\begin{aligned}
\bar{\Theta}^{1}(s, \theta) & =H_{1}\left[\Theta^{1}(r, \theta) ; r \rightarrow s\right] \\
& =\int_{1}^{\infty} \Theta^{1}(r, \theta)\left(r^{s-1}-r^{-s-1}\right) d r
\end{aligned}
$$

whose inverse is

$$
\begin{aligned}
\Theta^{1}(r, \theta) & =H_{1}^{-1}\left[\bar{\Theta}^{1}(s, \theta) ; s \rightarrow r\right] \\
& =\frac{1}{2 \pi i} \int_{\gamma-i \infty}^{\gamma+i \infty} \bar{\Theta}^{1}(s, \theta) r^{-s} d s, \\
|\operatorname{Re}(s)|=|\gamma|<1 . &
\end{aligned}
$$

Since

$$
\begin{aligned}
H_{1}\left[r \frac{\partial}{\partial r}\left(r \frac{\partial \Theta^{1}(r, \theta)}{\partial r}\right) ; \quad r \rightarrow s\right]=s^{2} H_{1}\left[\Theta^{1}(r, s) ; r \rightarrow s\right] & \\
& -2 \frac{\partial \Theta^{(1)}}{\partial r}(r, \theta), \quad|\operatorname{Res}|<1,
\end{aligned}
$$

using boundary condition (ii), the equation $\nabla^{2} \Theta^{1}=0$ transforms into

$$
\frac{d^{2} \bar{\Theta}^{1}(s, \theta)}{d \theta^{2}}+s^{2} \bar{\Theta}^{1}(s, \theta)=0, \quad|\operatorname{Re}(s)|<1
$$

whose general solution is 


$$
\bar{\Theta}^{1}(s, \theta)=s^{-1}[A(s) \cos s \theta+B(s) \sin s \theta],
$$

where $A(s)$ and $B(s)$ are arbitrary functions of the transform variable $s$. Applying condition (iii) we find that

$$
\bar{\Theta}^{1}(s, \theta)=s^{-1} A(s) \frac{\sin (\theta-\pi) s}{\sin \pi s}
$$

or

$$
\Theta^{1}(r, \theta)=H_{1}^{-1}\left[s^{-1} A(s) \frac{\sin (\theta-\pi) s}{\sin \pi s} ; s \rightarrow \tau\right]
$$

Thus, to satisfy condition (iv), $A(s)$ must satisfy the dual integral equations

$$
\begin{gathered}
H_{1}^{-1}\left[s^{-1} A(s) ; s \rightarrow r\right]=0, \quad c<r<\infty, \\
H_{1}^{-1}[A(s) \cot \pi s ; s \rightarrow r]=\frac{B q_{0} \sin \theta_{0}}{\kappa T_{0}}\left(r+r^{-1}\right), \\
1<r<c .
\end{gathered}
$$

Dual equations of this type have been discussed by Tweed [2] from who's work it follows that

$$
\Theta^{1}(r, 0)=H(c-r) \int_{r}^{c} p(t) d t, \quad r>1
$$

where $H(r)$ is the heaviside function, 


$$
A(s)=\int_{1}^{c} p(t)\left(t^{-s}-t^{s}\right) d t
$$

and

$$
\begin{aligned}
p(t)= & \frac{B q_{0} \sin \theta_{0}}{\pi \kappa T_{0} \sqrt{(c-t)(c t-1)}} \cdot \\
& \cdot \int_{1}^{c} \sqrt{(c-x)(c x-1)}\left\{\frac{1}{x-t}+\frac{1}{1-x t}\right\}\left\{1+\frac{1}{x^{2}}\right\} d x \\
= & \frac{B q_{0} \sin \theta_{0} \sqrt{c}}{2 \kappa T_{0} \sqrt{(c-t)(c t-1)}} \cdot\left\{c^{2}+1-2 c t-t^{-1}\left(c^{2}+1-2 c t^{-1}\right)\right\}
\end{aligned}
$$

It follows from equation [1.1] that

$$
\begin{aligned}
\Theta(r, 0) & =\frac{-B q_{0}}{\kappa T_{0}}\left(r+\tau^{-1}\right) \cos \theta_{0} \\
& -\frac{B q_{0} \sin \theta_{0}}{2 \kappa T_{0}} H(c-r) \int_{r}^{c} \frac{\left[c^{2}+1-2 c t-t^{-1}\left(c^{2}+1-2 c t^{-1}\right)\right] d t}{\sqrt{(c-t)\left(t-c^{-1}\right)}} \\
& =\frac{-B q_{0}}{\kappa T_{0}}\left(r+r^{-1}\right) \cos \theta_{0} \\
& -\frac{B q_{0} \sin \theta_{0}}{\kappa T_{0}}\left(1+r^{-1}\right) H\left[(c-r)\left(r-c^{-1}\right)\right] \quad \sqrt{(c-r)\left(r-c^{-1}\right)}
\end{aligned}
$$

To find an expression for $\Theta^{1}(r, \theta)$ we recall that the solution to the Dirichlet problem:

$$
\nabla^{2} V(x, y)=0, \quad-\infty<x<\infty, \quad y>0
$$

24 
(i) $V(x, y) \rightarrow 0$ as $\sqrt{x^{2}+y^{2}} \rightarrow \infty$,

(ii) $V(x, 0)=w(x), \quad-\infty<x<\infty$,

is given by

$$
V(x, y)=\operatorname{Im} \frac{1}{\pi} \int_{-\infty}^{\infty} \frac{w(t) d t}{t-z}
$$

and, therefore, in view of [1.15], we see that

$$
\begin{aligned}
\Theta^{1}(r, \theta) & =\frac{-B q_{0} \sin \theta_{0}}{\kappa T_{0}} \operatorname{Im}\left\{\frac{1}{\pi} \int_{c^{-1}}^{c} \frac{\sqrt{(c-t)\left(t-c^{-1}\right)}\left(1+t^{-1}\right) d t}{t-z}\right\} \\
& =\frac{-B q_{0} \sin \theta_{0}}{\kappa T_{0}} \operatorname{Im}\left\{(z+1)\left[\frac{\sqrt{(z-c)\left(z-c^{-1}\right)}}{z}-1\right]\right. \\
& \left.+\frac{1}{z}+\frac{c^{-1}+c}{2}\right\} \\
& =\frac{-B q_{0} \sin \theta_{0}}{\kappa T_{0}} \operatorname{Im}\left\{\sqrt{r_{1} \tau_{2}} e^{i\left(\frac{\theta_{1}+\theta_{2}}{2}\right)}-r e^{i \theta}\right. \\
& \left.+\tau^{-1} e^{-i \theta}-1+\frac{c+c^{-1}}{2}+\frac{\sqrt{r_{1} \tau_{2}}}{r} e^{i\left(\frac{\theta_{1}+\theta_{2}}{2}\right)-i \theta}\right\} \\
& =\frac{-B q_{0} \sin \theta_{0}}{\kappa T_{0}}\left\{\sqrt{r_{1} \tau_{2}} \sin \left(\frac{\theta_{1}+\theta_{2}}{2}\right)-\left(r+r^{-1}\right) \sin \theta\right. \\
& \left.+\frac{\sqrt{r_{1} \tau_{2}}}{r} \sin \left(\frac{\theta_{1}+\theta_{2}}{2}-\theta\right)\right\}
\end{aligned}
$$

where

$$
z=r e^{i \theta}, \quad z-c=r_{1} e^{i \theta_{1}}, \text { and } z-c^{-1}=r_{2} e^{i \theta_{2}} .
$$


Thus,

$$
\begin{aligned}
\Theta(r, \theta) & =\frac{-B q_{0} \sin \theta_{0}}{\kappa T_{0}}\left(r+r^{-1}\right) \cos \theta \cos \theta_{0} \\
& -\frac{B q_{0} \sin \theta_{0}}{\kappa T_{0}} \sqrt{r_{1} r_{2}}\left\{\sin \left(\frac{\theta_{1}+\theta_{2}}{2}\right)\right. \\
& \left.+\frac{1}{r} \sin \left(\frac{\theta_{1}+\theta_{2}}{2}-\theta\right)\right\} .
\end{aligned}
$$




\section{The Mechanical Field}

We next investigate the mechanical part of the solution. We assume that the stress function is of the form

$$
\phi(r, \theta)=\phi^{0}(r, \theta)+\hat{\phi}(r, \theta)
$$

Because of symmetry and linearity of the problem conditions 1,3 , and $4 \mathrm{~b}$ will be satisfied if $\hat{\phi}(r, \theta)$ is the result of superimposing the solutions of the following two problems [4]:

\section{Problem 1.1}

Solve the equations of plane strain linear isothermal elasticity in the region $r>1,0<\theta<\pi$ subject to the conditions:

(i) At infinity $\phi^{\prime}(r, \theta)=0(r)$,

(ii) $\hat{u}^{\prime}(r, 0)=\ddot{u}^{\prime}(r, \pi)=0, \quad 1<r<\infty$,

(iii) $s_{\theta \theta}^{\prime}(r, 0)=s_{\theta \theta}^{\prime}(r, \pi)=0, \quad 1<r<\infty$.

\section{Problem 1.2}

Solve the equations of plane strain linear thermoelasticity in the region $0 \leq r<\infty, 0<\theta<\pi$ for the case where

(i) At infinity $\phi^{\prime \prime}(r, \theta)=0\left(r^{-2}\right)$,

(ii) $\left.u^{\prime \prime}(r, \theta), v^{\prime \prime} r, \theta\right), s_{i j}^{\prime \prime}(r, \theta)$ are all bounded at the origin,

(iii) $s_{\theta \theta}^{\prime \prime}(r, 0)=s_{\theta \theta}^{\prime \prime}(r, \pi)=0, \quad 0<r<\infty$,

(iv) $u^{\prime \prime}(r, \pi)=0, \quad 0<r<\infty$,

(v) $\Theta^{\prime \prime}(r, 0)=\Theta^{1}(r, 0), \quad r>0$ $\Theta^{\prime \prime}(r, \pi)=0, \quad r>0$.

It is easy to show that the Michell solution to problem 1.1 is given by: 


$$
\begin{gathered}
\phi^{\prime}(r, \theta)=\sum_{n=1}^{\infty}\left\{b_{n} r^{-n}+d_{n} r^{2-n}\right\} \sin n \theta, \\
s_{r r}^{\prime}(r, \theta)=-\sum_{n=1}^{\infty}\left\{n(n+1) b_{n} r^{-n-2}+(n-1)(n+2) d_{n} r^{-n}\right\} \sin n \theta, \\
s_{r \theta}^{\prime}(r, \theta)=\sum_{n=1}^{\infty}\left\{n(n+1) b_{n} r^{-n-2}+n(n-1) d_{n} r^{-n}\right\} \cos n \theta, \\
s_{\theta \theta}^{\prime}(r, \theta)=\sum_{n=1}^{\infty}\left\{n(n+1) b_{n} r^{-n-2}+(n-1)(n-2) d_{n} r^{-n}\right\} \sin n \theta, \\
v^{\prime}(r, \theta)=-b_{1} r^{-2} \cos \theta \\
u_{n=1}^{\prime}\{r, \theta)=b_{1} r^{-2} \sin \theta \\
\sum_{n=1}^{\infty}\left\{n b_{n} r^{-n-1}+d_{n}[n+2-4 \nu] r^{-n+1}\right\} \sin n \theta, \\
\end{gathered}
$$

We use Mellin transforms to solve problem 1.2. From Chapter 2, we have the solution: 


$$
\begin{aligned}
& \bar{\phi}^{\prime \prime}(s, \theta)=\bar{A}(s) \sin s \theta+\bar{B}(s) \cos s \theta \\
& +\bar{C}(s) \sin (s+2) \theta+\bar{D}(s) \cos (s+2) \theta \\
& \overline{r^{2} s_{r r}^{\prime \prime}}(s, \theta)=\frac{\partial^{2} \bar{\phi}^{\prime \prime}(s, \theta)}{\partial \theta^{2}}-s \bar{\phi}^{\prime \prime}(s, \theta) \\
& \overline{r^{2} s_{r \theta}^{\prime \prime}}(s, \theta)=(s+1) \frac{\partial \bar{\phi}^{\prime \prime}(s, \theta)}{\partial \theta} \\
& \overline{r^{2} s_{\theta \theta}^{\prime \prime}}(s, \theta)=s(s+1) \bar{\phi}^{\prime \prime}(s, \theta) \text {, } \\
& \overline{r u^{\prime \prime}}(s, \theta)=-(s+1)^{-1}\left\{(1-\nu) \frac{\partial^{2} \bar{\phi}^{\prime \prime}(s, \theta)}{\partial \theta^{2}}-s(1+\nu s) \bar{\phi}^{\prime \prime}(s, \theta)\right\} \\
& -\frac{E \alpha T_{0}}{p_{0}}(s+1)^{-1} \quad \bar{\Theta}^{1}(s+2, \theta) \\
& \overline{r v^{\prime \prime}}(s, \theta)=-(s+1)^{-1}(s+2)^{-1}\left\{(1-\nu) \frac{\partial^{3} \bar{\phi}^{\prime \prime}(s, \theta)}{\partial \theta^{3}}\right. \\
& \left.+\left[(1-\nu) s^{2}+(s+1)(s+2)\right] \frac{\partial \bar{\phi}(s, \theta)}{\partial \theta}\right\} \\
& -\frac{E \alpha T_{0}}{p_{0}}(s+1)^{-1}(s+2)^{-1} \frac{\partial \bar{\Theta}^{1}(s+2, \theta)}{\partial \theta} \text {. }
\end{aligned}
$$

Condition (iii) implies that 


$$
\bar{B}(s)=-\bar{D}(s) \text { and } \bar{A}(s)=-\bar{C}(s)
$$

Thus,

$$
\begin{aligned}
\bar{\phi}^{\prime \prime}(s, \theta) & =\bar{A}(s)[\sin s \theta-\sin (s+2) \theta] \\
& +\bar{B}(s)[\cos s \theta-\cos (s+2) \theta]
\end{aligned}
$$

and condition (iv) implies that

$$
\bar{A}(s)=-\bar{B}(s) \cot \pi s .
$$

This gives the solution

$$
\bar{\phi}^{\prime \prime}(s, \theta)=\frac{\bar{B}(s)}{\sin \pi s}\{\sin (s+2)(\theta-\pi)-\sin s(\theta-\pi)\},
$$

$$
\overline{r^{2} s_{r r}^{\prime \prime}}(s, \theta)=\frac{(s+1) \bar{B}(s)}{\sin \pi s}\{s \sin s(\theta-\pi)-(s+4) \sin (s+2)(\theta-\pi)\}
$$

$$
\overline{r^{2} s_{r \theta}^{\prime \prime}}(s, \theta)=\frac{(s+1) \bar{B}(s)}{\sin \pi s}\{(s+2) \cos (s+2)(\theta-\pi)-s \cos s(\theta-\pi)\},
$$

$$
\overline{r^{2} s_{\theta \theta}^{\prime \prime}}(s, \theta)=\frac{s(s+1) \bar{B}(s)}{\sin \pi s}\{\sin (s+2)(\theta-\pi)-\sin s(\theta-\pi)\},
$$

$$
\begin{aligned}
\overline{r u^{\prime \prime}}(s, \theta) & =(s+1)^{-1}\left\{\frac{(s+1) \bar{B}(s)}{\sin \pi s)}[(s+4-4 \nu) \sin (s+2)(\theta-\pi)\right. \\
& -s \sin s(\theta-\pi)]\}-\frac{E \alpha T_{0}}{p_{0}} r^{-1} \frac{\bar{\Theta}^{\prime \prime}(s+2, \theta)}{s+1}
\end{aligned}
$$




$$
\begin{aligned}
\overline{r v^{\prime \prime}}(s, \theta) & =(s+1)^{-1}\left\{\frac{(s+1) \bar{B}(s)}{\sin \pi s}[s \cos s(\theta-\pi)\right. \\
& -(s-2+4 \nu) \cos (s+2)(\theta-\pi)]\} \\
& -\frac{E \alpha T_{0}}{p_{0}} r^{-1} \frac{\partial \bar{\Theta}^{1}(s+2, \theta)}{\partial \theta} \cdot(s+1)^{-1}(s+2)^{-1}
\end{aligned}
$$

and we see that to satisfy conditions (i) and (ii) we must have $-2<\operatorname{Re}(s)<-1$. On setting $\bar{B}(s)=\frac{1}{2}(s+1)^{-1} A(s)$, this leads, via superposition, to the stress function

$$
\begin{aligned}
\phi(r, \theta)= & \phi^{0}(r, \theta)+\sum_{n=1}^{\infty}\left[b_{n} r^{-n}+d_{n} r^{2-n}\right] \sin n \theta \\
& +M^{-1}\left[\frac{A(s)}{2(s+1) \sin \pi s}\{\sin (s+2)(\theta-\pi)\right. \\
& -\sin s(\theta-\pi)\} ; r], \quad-2<\operatorname{Re}(s)<-1,
\end{aligned}
$$

and, hence, the stresses are given by:

$$
\begin{aligned}
s_{r r}(r, \theta) & =\left(r^{-1}-r^{-3}\right) \cos \left(\theta-\theta_{0}\right) \\
& -\sum_{n=1}^{\infty}\left\{n(n+1) b_{n} r^{-n-2}+(n-1)(n+2) d_{n} r^{-n}\right\} \sin n \theta \\
& +r^{-2} M^{-1}\left[\frac{A(s)}{2 \sin \pi s}\{s \sin (\theta-\pi) s\right. \\
& -(s+4) \sin (\theta-\pi)(s+2)\} ; r]
\end{aligned}
$$




$$
\begin{aligned}
s_{r \theta}(r, \theta) & =\left(r^{-1}-r^{-3}\right) \sin \left(\theta-\theta_{0}\right) \\
& +\sum_{n=1}^{\infty}\left\{n(n+1) b_{n} r^{-n-2}+n(n-1) d_{n} r^{-n}\right\} \cos n \theta \\
& +r^{-2} M^{-1}\left[\frac{A(s)}{2 \sin \pi s}\{(s+2) \cos (s+2)(\theta-\pi)\right. \\
& -s \cos s(\theta-\pi)\} ; r]
\end{aligned}
$$

$$
\begin{aligned}
s_{\theta \theta}(r, \theta) & =\left(r^{-1}+r^{-3}\right) \cos \left(\theta-\theta_{0}\right) \\
& +\sum_{n=1}^{\infty}\left\{n(n+1) b_{n} r^{-n-2}+(n-1)(n-2) d_{n} r^{-n}\right\} \sin n \theta \\
& +r^{-2} M^{-1}\left[\frac{s A(s)}{2 \sin \pi s}\{\sin (s+2)(\theta-\pi)\right. \\
& -\sin s(\theta-\pi)\} ; r]
\end{aligned}
$$

and the displacements are: 


$$
\begin{aligned}
& u(r, \theta)=\left\{\frac{1}{2 r^{2}}+(1-2 \nu) \ln r-2(1-\nu)\right\} \cos \left(\theta-\theta_{0}\right) \\
& -(1-\nu)\left\{r^{2}+\ln r^{2}-2\right\} \cos \left(\theta-\theta_{0}\right)+b_{1} r^{-2} \sin \theta \\
& +\sum_{n=2}^{\infty}\left\{n b_{n} r^{-n-1}+d_{n}[n+2-4 \nu] r^{-n+1}\right\} \sin n \theta \\
& +r^{-1} M^{-1}\left[\frac{A(s)}{2(s+1) \sin \pi s}\{(s+4-4 \nu) \sin (s+2)(\theta-\pi)\right. \\
& -s \sin s(\theta-\pi)\} ; r] \\
& -\frac{E \alpha T_{0}}{p_{0}} r^{-1} M^{-1}\left[\frac{\bar{\Theta}^{1}(s+2, \theta)}{s+1} ; r\right] \\
& v(r, \theta)=\left\{\frac{1}{2 r^{2}}+(1-2 \nu)[1-\ln r]\right\} \sin \left(\theta-\theta_{0}\right) \\
& -(1-\nu)\left\{r^{2}+2-\ln r^{2}\right\} \sin \left(\theta-\theta_{0}\right)-b_{1} \tau^{-2} \cos \theta \\
& -\sum_{n=2}^{\infty}\left\{n b_{n} r^{-n-1}+d_{n}[n-4+4 \nu] r^{-n+1}\right\} \cos n \theta \\
& +r^{-1} M^{-1}\left[\frac{A(s)}{2(s+1) \sin \pi s}\{s \cos s(\theta-\pi)\right. \\
& -(s-2+4 \nu) \cos (s+2)(\theta-\pi)\} ; r] \\
& -\frac{E \alpha T_{0}}{p_{0}} r^{-1} M^{-1}\left[\frac{1}{(s+1)(s+2)} \frac{\partial \bar{\Theta}^{1}(s+2, \theta)}{\partial \theta} ; r\right] \text {, }
\end{aligned}
$$

where $-2<\operatorname{Re}(s)<-1$ and

$$
\bar{\Theta}^{1}(\sigma, \theta)=M\left[\Theta^{1}(r, \theta) ; r \rightarrow \sigma\right], \quad 0<\operatorname{Re}(\sigma)<1
$$

33 
Applying condition 4, we find that

$$
A(s)=-\frac{E \alpha T_{0}}{2(1-\nu) p_{0}} \bar{\Theta}^{1}(s+2,0)
$$

and hence, in view of [2.1.18] and [1.15],

$$
A(s)=\sin \theta_{0} \int_{c^{-1}}^{c} Q(t) t^{s+2} d t
$$

where $0<\operatorname{Re}(s+2)<1, \quad$ and

$$
Q(t)=t^{-2}(t+1) \sqrt{(c-t)\left(t-c^{-1}\right)}
$$

Similarly, on applying condition 2 , we find that

$$
\begin{aligned}
& \sum_{n=1}^{\infty}\left\{n(n+1) b_{n}+(n-1)(n+2) d_{n}\right\} \sin n \theta \\
& =M^{-1}\left[\frac{A(s)}{2 \sin \pi s}\{s \sin s(\theta-\pi)\right. \\
& -(s+4) \sin (s+2)(\theta-\pi)\} ; 1]
\end{aligned}
$$

and

$$
\begin{aligned}
& \sum_{n=1}^{\infty}\left\{n(n+1) b_{n}+n(n-1) d_{n}\right\} \cos n \theta \\
& =M^{-1}\left[\frac{A(s)}{2 \sin \pi s}\{s \cos s(\theta-\pi)\right. \\
& -(s+2) \cos (s+2)(\theta-\pi)\} ; 1]
\end{aligned}
$$


where $0<\theta<\pi, \quad 0<\operatorname{Re}(s+2)<1$.

In $[2.31]$ and $[2.32]$ the Fourier coefficients are clearly given by

$$
\begin{aligned}
n(n+1) b_{n} & +(n-1)(n+2) d_{n} \\
& =\frac{2}{\pi} M^{-1}\left[\frac { A ( s ) } { 2 \operatorname { s i n } \pi s } \left\{s \int_{0}^{\pi} \sin s(\theta-\pi) \sin n \theta d \theta\right.\right. \\
& \left.\left.-(s+4) \int_{0}^{\pi} \sin (s+2)(\theta-\pi) \sin n \theta d \theta\right\} ; 1\right]
\end{aligned}
$$

and

$$
\begin{aligned}
n(n+1) b_{n} & +n(n-1) d_{n} \\
& =\frac{2}{\pi} M^{-1}\left[\frac { A ( s ) } { 2 \operatorname { s i n } \pi s } \left\{s \int_{0}^{\pi} \cos s(\theta-\pi) \cos n \theta d \theta\right.\right. \\
& \left.\left.-(s+2) \int_{0}^{\pi} \cos (s+2)(\theta-\pi) \cos n \theta d \theta\right\} ; 1\right]
\end{aligned}
$$

Therefore, on making use of the identities

$$
\begin{aligned}
& \frac{2}{\pi} \int_{0}^{\pi} \sin \{(\theta-\pi)(s+k)\} \sin n \theta d \theta \\
& =\frac{(-1)^{k}}{\pi}\left\{\frac{1}{s+k-n}-\frac{1}{s+k+n}\right\} \sin \pi s \\
& \frac{2}{\pi} \int_{0}^{\pi} \cos \{(\theta-\pi)(s+k)\} \cos n \theta d \theta \\
& =\frac{(-1)^{k}}{\pi}\left\{\frac{1}{s+k-n}+\frac{1}{s+k+n}\right\} \sin \pi s
\end{aligned}
$$

35 
and

$\frac{1}{\pi} \int_{0}^{\pi} \cos (\theta-\pi)(s+k) d \theta=\frac{(-1)^{k}}{\pi} \frac{\sin \pi s}{s+k}, \quad k \quad$ any integer

we find that $b_{n}$ and $d_{n}$ must satisfy the equations:

$$
\begin{aligned}
n(n+1) b_{n} & +(n-1)(n+2) d_{n} \\
& =\frac{1}{2 \pi} M^{-1}\left[A ( s ) \left\{\frac{n}{s-n}+\frac{n}{s+n}\right.\right. \\
& \left.\left.-\frac{n+2}{s+2-n}-\frac{n-1}{s+2+n}\right\} ; 1\right],
\end{aligned}
$$

$$
\begin{aligned}
n(n+1) b_{n} & +n(n-1) d_{n} \\
& =\frac{1}{2 \pi} M^{-1}\left[A ( s ) \left\{\frac{n}{s-n}-\frac{n}{s+n}\right.\right. \\
& \left.\left.+\frac{n}{s+2+n}-\frac{n}{s+2-n}\right\} ; 1\right],
\end{aligned}
$$

$0<\theta<\pi, \quad 0<\operatorname{Re}(s+2)<1$. It follows that

$$
\begin{aligned}
n(n+1) b_{n} & =\frac{1}{2 \pi} M^{-1}\left[A ( s ) \left\{\frac{n}{s-n}\right.\right. \\
& \left.\left.-\frac{n(n+1)}{s+n}+\frac{n^{2}}{s+2+n}\right\} ; 1\right]
\end{aligned}
$$

and 


$$
\begin{aligned}
n(n-1) d_{n} & =\frac{1}{2 \pi} M^{-1}\left[A ( s ) \left\{\frac{n^{2}}{s+n}\right.\right. \\
& \left.\left.-\frac{n(n-1)}{s+2+n}+\frac{n}{s+2-n}\right\} ; 1\right]
\end{aligned}
$$

Now, by (2.29),

$$
\begin{array}{rlrl}
A(s) & =\sin \theta_{0} \int_{c^{-1}}^{c} Q(t) t^{s+2} d t & \\
& =\sin \theta_{0} \int_{c^{-2}}^{c} Q(t) t^{-(s+2)} d t, & & -2<\operatorname{Re}(s)<-1, \\
& =\sin \theta_{0} \int_{c^{-1}}^{c} Q(t) t^{-\sigma} d t, & & 0<\operatorname{Re}(\sigma)<1,
\end{array}
$$

where

$$
Q(t)=\frac{t+1}{t^{2}} \sqrt{(c-t)\left(t-c^{-1}\right)} \quad \text { and } \quad \sigma=s+2
$$

Therefore,

$$
\begin{array}{r}
n(n+1) b_{n}=\frac{\sin \theta_{0}}{2 \pi} \int_{c^{-1}}^{c} Q(t) M^{-1}\left[\frac{n}{\sigma-n-2}\right. \\
\left.-\frac{n(n+1)}{\sigma+n-2}+\frac{n^{2}}{\sigma+n} ; \sigma \rightarrow t\right] d t
\end{array}
$$

and

$$
\begin{array}{r}
n(n-1) d_{n}=\frac{\sin \theta_{0}}{2 \pi} \int_{c^{-1}}^{c} Q(t) M^{-1}\left[\frac{n^{2}}{\sigma+n-2}\right. \\
\left.-\frac{n(n-1)}{\sigma+n}-\frac{n}{\sigma-n} ; \sigma \rightarrow t\right] d t,
\end{array}
$$

37 
$0<\operatorname{Re}(\sigma)<1, n \geq 1$. It follows that

$$
2 b_{1}=\frac{\sin \theta_{0}}{2 \pi} \int_{1}^{c} Q(t)\left\{3 t^{-1}-t^{-3}\right\} d t
$$

and, for $n \geq 2$,

$$
\begin{aligned}
n(n+1) b_{n} & =\frac{\sin \theta_{0}}{2 \pi} \int_{1}^{c} Q(t)\left\{n^{2} t^{-n}-n(n+1) t^{-n+2}\right. \\
& \left.-n t^{-n-2}\right\} d t
\end{aligned}
$$

and

$$
n(n-1) d_{n}=\frac{\sin \theta_{0}}{2 \pi} \int_{1}^{c} Q(t)\left\{n^{2} t^{-n+2}-n(n-2) t^{-n}\right\} d t
$$

We are now in the position to calculate the quantities $f_{1}(r)$ and $f_{2}(r)$ as required. From equation [2.24]

$$
\begin{aligned}
f_{1}(r) & =s_{\theta \theta}(r, 0) \sec \theta_{0} \\
& =r^{-1}+r^{-3} .
\end{aligned}
$$

In contrast (2.23) yields

$$
\begin{aligned}
f_{2}(r) & =r^{-3}-r^{-1}+r^{-2} \csc \theta_{0} M^{-1}[A(s) \cot \pi s ; r] \\
& +\csc \theta_{0} \sum_{n=1}^{\infty}\left\{n(n+1) b_{n} r^{-n-2}+n(n-1) d_{n}\right\}
\end{aligned}
$$


$0<\operatorname{Re}(s+2)<1$, which needs to be reduced further. On substituting for $A(s)$ from equation [2.37] and using the result

$$
M^{-1}[\pi \cot n \pi s ; \quad x]=\frac{1}{1-x}, \quad 0<\operatorname{Re}(s)<1
$$

we obtain

$$
\begin{aligned}
r^{-2} M^{-1}[A(s) \cot \pi s ; r] & =\frac{\sin \theta_{0}}{\pi} \int_{c^{-1}}^{c} Q(t) \frac{d t}{1-r t} \\
& =\frac{\sin \theta_{0}}{\pi} \int_{1}^{c} Q(t)\left[\frac{1}{1-r t}+\frac{t}{t-r}\right] d t
\end{aligned}
$$

Therefore,

$$
\begin{aligned}
\sum_{n=1}^{\infty}\left\{n(n+1) b_{n} \tau^{-n-2}+n(n-1) d_{n} \tau^{-n}\right\} \\
\quad=\frac{\sin \theta_{0}}{2 \pi} \int_{1}^{c} Q(t)\left\{3 t^{-1}-t^{-3}\right\} r^{-3} d t \\
+\frac{\sin \theta_{0}}{2 \pi} \int_{1}^{c} Q(t) \sum_{n=2}^{\infty}\left\{n^{2} t^{-n}-n(n+1) t^{-n+2}-n t^{-n-2}\right\} r^{-n-2} d t \\
+\frac{\sin \theta_{0}}{2 \pi} \int_{1}^{c} Q(t) \sum_{n=2}^{\infty}\left\{n^{2} t^{-n+2}-n(n-2) t^{-n}\right\} r^{-n} d t \\
=\frac{\sin \theta_{0}}{2 \pi} \int_{1}^{c} Q(t)\left\{\frac{3}{t r^{3}}-\sum_{1}^{\infty} n(r t)^{-n-2}+t^{2} \sum_{2}^{\infty} n^{2}(r t)^{-n-2}\right. \\
\left.-t^{4} \sum_{n=2}^{\infty} n(n+1)(r t)^{-n-2}+t^{2} \sum_{2}^{\infty} n^{2}(r t)^{-n}-\sum_{n=2}^{\infty} n(n-2)(r t)^{-n}\right\} d t \\
=\frac{\sin \theta_{0}}{\pi} \int_{1}^{c} Q(t)\left\{\frac{\left(t^{2}-1\right)^{2}}{(1-r t)^{3}}+\frac{\left(t^{2}-1\right)}{(1-r t)^{2}}\right. \\
\left.\left.\quad-\frac{\left(t^{2}+1\right)}{1-r t}-\frac{\left(t^{2}+1\right)}{r t}+\frac{t^{2}+1}{r^{3} t}\right\}^{2} d t .49\right)
\end{aligned}
$$


See Appendix A for details. Thus, [2.46], [2.48] and [2.49] imply that

$$
\begin{aligned}
f_{2}(r) & =r^{-3}-r^{-1}+\frac{1}{\pi} \int_{1}^{c} Q(t)\left\{\frac{t}{t-r}+\frac{\left(t^{2}-1\right)^{2}}{(1-r t)^{3}}+\frac{t^{2}-1}{(1-r t)^{2}}\right\} d t \\
& -\frac{1}{\pi} \int_{1}^{c} M(t)\left\{\frac{t^{2}}{1-r t}+\frac{t^{2}+1}{r t}-\frac{t^{2}+1}{r^{3} t}\right\} d t \\
& =\left(r^{-3}-r^{-1}\right)\left\{1+\frac{1}{8}\left(c-c^{-1}\right)^{2}+\frac{1}{2}\left(c^{\frac{1}{2}}-c^{-\frac{1}{2}}\right)^{2}\right\} \\
& +\left[\frac{1}{2}\left(c^{\frac{1}{2}}-c^{-} \frac{1}{2}\right)-r+\frac{1}{r}\right] \\
& +\frac{1}{\pi} \int_{1}^{c} \Delta(t) \frac{1+t}{t^{2}}\left\{\frac{\left(t^{2}-2\right)^{2}}{(1-r t)^{3}}+\frac{t^{2}-1}{(1-r t)^{2}}-\frac{t^{2}+1}{1-r t}\right\} d t \\
& =\left(r^{-3}-r^{-1}\right)\left\{1+\frac{1}{8}\left(c-c^{-1}\right)^{2}+\frac{1}{2}\left(c^{\frac{1}{2}}-c^{\left.-\frac{1}{2}\right)^{2}}\right\}\right. \\
& +\left[\frac{1}{2}\left(c^{\frac{1}{2}}-c^{-\frac{1}{2}}\right)-r+\frac{1}{r}\right]+I I(r) \\
\Delta(t) & =\sqrt{(c-t)\left(t-c^{-1}\right) .}
\end{aligned}
$$

$I I(r) \quad$ is evaluated in appendix $\mathrm{B}$. 


\section{Chapter 5}

\section{Solution of Problem 2}

Problem 2 may be solved by the Mellin transform technique of Tweed and Rooke [4]. In which case it can be shown [5] that the solution is such that for $1<r<c$,

$$
u(r, 0)-u(r, 2 \pi)=-4(1-\nu) \sin \theta_{0} \int_{r}^{c} \frac{P_{2}(t) d t}{\sqrt{(c-t)(t-1)}},
$$

and

$$
v(r, 0)-v(r, 2 \pi)=-4(1-\nu) \cos \theta_{0} \int_{r}^{c} \frac{P_{1}(t) d t}{\sqrt{(c-t)(t-1)}}
$$

where the quantities $P_{i}(t), i=1,2$ must satisfy the singular integral equations

$$
\frac{1}{\pi} \int_{1}^{c} \frac{P_{i}(t)}{\sqrt{(c-t)(t-1)}}\left\{\frac{1}{t-r}+K_{i}(r, t)\right\} d t=-f_{i}(r)
$$

$1<r<c$, with subsidiary conditions

$$
P_{i}(1)=0, \quad i=1,2
$$

and kernel functions

$$
K_{1}(r, t)=\frac{\left(1-t^{2}\right)^{2}}{t(1-t r)^{3}}-\frac{t\left(1-t^{2}\right)}{(1-r t)^{2}}-\frac{t}{(1-r t)}+\frac{1-t^{2}}{r^{2} t}
$$


and

$$
K_{2}(r, t)=\frac{\left(1-t^{2}\right)^{2}}{t(1-r t)^{3}}-\frac{\left(1-t^{2}\right)}{t(1-r t)^{2}}-\frac{t}{(1-r t)}
$$

respectively. Since $f_{i}(r)$ are known, equations [1.3] maybe solved numerically and the results used to calculate the stress intensity factors and crack formation energy which are quantities of interest to workers in fracture mechanics.

The opening and sliding mode stress intensity factors $k_{1}$ and $k_{2}$, respectively, and the crack formation energy $W$ are defined by the equations

$$
\begin{aligned}
& k_{1}=-\frac{E}{4\left(1-\nu^{2}\right)} \lim _{R \rightarrow C^{-}} \sqrt{2(C-R)} \frac{\partial}{\partial R}\left[U_{\theta}(R, 0)-U_{\theta}(R, 2 \pi)\right] \\
& k_{2}=-\frac{E}{4\left(1-\nu^{2}\right)} \lim _{R \rightarrow C^{-}} \sqrt{2(C-R)} \frac{\partial}{\partial R}\left[U_{R}(R, 0)-U_{R}(R, 2 \pi)\right]
\end{aligned}
$$

and

$$
\begin{aligned}
W & =\frac{\cos \theta_{0}}{2} \int_{B}^{C} p_{0} f_{1}(R / B)\left[U_{\theta}(R, 0)-U_{\theta}(R, 2 \pi)\right] d R \\
& +\frac{\sin \theta_{0}}{2} \int_{B}^{C} p_{0} f_{2}(R / B)\left[U_{R}(R, 0)-U_{R}(R, 2 \pi)\right] d R .
\end{aligned}
$$

It is well known [6] that the stress intensity factor $k_{0}$ and the crack formation energy $W_{0}$ of a crack of length $2(C-B)$ in an infinite elastic solid which is subject to a uniform all round tension $p_{0}$ are given by $k_{0}=$ $p_{0}(C-B)^{\frac{1}{2}}$ and $W_{0}=\pi\left(1-\nu^{2}\right) p_{0}^{2}(C-B)^{2} / E$, respectively. Therefore, upon substituting $[1.1]-[1.2]$ into $[1.7]-[1.9]$ we have 


$$
\begin{aligned}
& \frac{k_{1}}{k_{0}}=\frac{-\sqrt{2}}{(c-1)} P_{1}(c) \cos \theta_{0}, \\
& \frac{k_{2}}{k_{0}}=\frac{-\sqrt{2}}{(c-1)} P_{2}(c) \sin \theta_{0}
\end{aligned}
$$

and

$$
\frac{W}{W_{0}}=\frac{W_{1}}{W_{0}} \cos \theta_{0}+\frac{W_{2}}{W_{0}} \sin \theta_{0}
$$

where

$$
\begin{aligned}
& \frac{W_{1}}{W_{0}}=\frac{-2}{\pi(c-1)^{2}} \int_{1}^{c} \frac{P_{1}(t)}{\sqrt{(c-t)(t-1)}} \int_{1}^{t} f_{1}(r) d r d t \\
& \frac{W_{2}}{W_{0}}=\frac{-2}{\pi(c-1)^{2}} \int_{1}^{c} \frac{P_{2}(t)}{\sqrt{(c-t)(t-1)}} \int_{1}^{t} f_{2}(r) d r d t .
\end{aligned}
$$




\section{CHAPTER 6}

\section{NUMERICAL PROCEDURES}

\section{The Stress Intensities and The Crack Formation Energies}

Following Erdogan and Gupta [11] we set

$x_{\kappa}=\cos \frac{(2 k-1) \pi}{2 n}, \quad t_{k}=\frac{1}{2}(c-1) x_{k}+\frac{1}{2}(c+1)$,

$r_{j}=\frac{1}{2}(c-1) \cos \frac{j \pi}{n}+\frac{1}{2}(c+1), \quad k=1,2, \ldots, n, \quad j=1,2, \ldots, n-1$, and replace the singular integral equations $[5.1 .3]$ by the linear algebraic system

$$
\begin{aligned}
& \frac{1}{n} \sum_{k=1}^{n} P_{i}\left(t_{k}\right)\left\{\frac{1}{t_{k}-r_{j}}+K_{i}\left(r_{j}, t_{k}\right)\right\}=-f_{i}\left(r_{j}\right), \\
& \frac{1}{n} \sum_{k=1}^{n}(-1)^{k} P_{i}\left(t_{k}\right)\left(\frac{1-x_{k}}{1+x_{k}}\right)^{\frac{1}{2}}=0,
\end{aligned}
$$

$j=1,2, \ldots, n-1 ; i=1,2$. Having solved the system for the $P_{i}\left(t_{k}\right)$ we calculate $k_{i} / k_{0}$ and from the Chebyshev-Lagrange interpolation formula

$$
\frac{k_{i}}{k_{0}}=\frac{\sqrt{2}}{n(c-1)} \sum_{k=1}^{n}(-1)^{k}\left(\frac{1+x_{k}}{1-x_{k}}\right)^{\frac{1}{2}} P_{i}\left(t_{k}\right)
$$

and $W_{1} / W_{0}$ from the Gaussian quadrature formula

$$
\frac{W_{i}}{W_{0}}=\frac{-2}{n(c-1)} \sum_{k=1}^{n} P_{i}\left(t_{k}\right) \int_{1}^{t_{k}} f_{i}(r) d r
$$

$i=1,2$, as described by Tweed and Rooke [5]. 


\section{The Computation of the Energy Weight Functions}

By [4.2.45]

$$
\begin{aligned}
\int_{1}^{t} f_{1}(r) d r & =\int_{1}^{t}\left(r^{-1}+r^{-2}\right) d r \\
& =\ln t+\frac{1}{2}\left(1-t^{-2}\right) .
\end{aligned}
$$

Similarly, by [4.2.50] we have

$$
\begin{aligned}
& \int_{1}^{t} f_{2}(r) d r=\left[1+\frac{1}{\pi} \int_{1}^{c} \Delta(\rho) \rho^{-3}(\rho+1)\left(\rho^{2}+1\right) d \rho\right] \int_{1}^{t}\left(r^{-3}-r^{-1}\right) d r \\
& +\frac{1}{\pi} \int_{1}^{t} \int_{1}^{c} \Delta(\rho) \frac{1+\rho}{\rho^{2}}\left\{\frac{\rho}{\rho-r}+\frac{1}{1-r \rho}\right\} d \rho d r \\
& +\frac{1}{\pi} \int_{1}^{t} \int_{1}^{c} \Delta(\rho) \frac{1+\rho}{\rho^{2}}\left\{\frac{\left(\rho^{2}-1\right)^{2}}{(1-\tau \rho)^{3}}+\frac{\rho^{2}-1}{(1-r \rho)^{2}}-\frac{\rho^{2}+1}{1-r \rho}\right\} d \rho d r \\
& =\left\{1+\frac{1}{8}\left(c-c^{-1}\right)^{2}+\frac{1}{2}\left(c^{\frac{1}{2}}-c^{-\frac{1}{2}}\right)^{2}\right\}\left\{\frac{1}{2}\left(1-t^{-2}\right)-\ln t\right\} \\
& +\left\{\frac{1}{2}\left(c^{\frac{1}{2}}-c^{-\frac{1}{2}}\right)(t-1)-\frac{1}{2}\left(t^{2}-1\right)+\ln t\right\} \\
& +\frac{1}{\pi} \int_{1}^{c} \Delta(\rho) \frac{1+\rho}{\rho^{2}}\left\{\frac{\left(\rho^{2}-1\right)^{2}}{2 \rho} \cdot \frac{1}{(1-\rho t)^{2}}+\frac{\rho^{2}-1}{\rho(1-\rho t)}\right. \\
& \left.+\frac{1-\rho^{2}}{2 \rho}+\frac{\rho^{2}+1}{\rho} \ln \left|\frac{\rho t-1}{\rho-1}\right|\right\} d \rho \\
& =\left\{1+\frac{1}{8}\left(c-c^{-1}\right)^{2}+\frac{1}{2}\left(c^{\frac{1}{2}}-c^{-\frac{1}{2}}\right)^{2}\right\}\left\{\frac{1}{2}\left(1-t^{-2}\right)-\ln t\right\} \\
& +\left\{\frac{1}{2}\left(c^{\frac{1}{2}}-c^{-\frac{1}{2}}\right)^{2}(t-1)-\frac{1}{2}\left(t^{2}-1\right)+\ln t\right\}+I I I(t) .
\end{aligned}
$$


$I I I(t)$ is evaluated in Appendix B.

\section{Numerical Results}

The results of the computations described above are displayed in figure 2 which shows the variation of $k_{1} /\left(k_{0} \cos \theta_{0}\right),-k_{2} /\left(k_{0} \sin \theta_{0}\right), W_{1} / W_{0}$, and $W_{2} / W_{0}$ with the relative crack length $L=(C-B) / B$.

When $L<<1, f_{1}(r) \approx 2$ and $f_{2}(r)=0$ so that the crack behaves like an edge crack in a half-space under uniaxial tension $2 p_{0} \cos \theta_{0}$. Numerically we find that as $L$ approaches zero $k_{1}$ and $k_{2}$ approach the expected values $1.12\left(2 p_{0} \cos \theta_{0}\right)(C-B)^{\frac{2}{2}}$ and zero, respectively.

Similarly, when $L>>1$, the crack and the hole behave like a line crack of length $C+B$ in a uniform heat flow

$$
g=q_{0}\left(\cos \theta_{0} i+\sin \theta_{0} j\right)
$$

Again we find numerically that $k_{1}$ and $k_{2}$ approach their theoretical limiting values [12] of zero and $-\left(p_{0} / 8 B\right) \sqrt{2} \sin \theta_{0}(C+B)^{\frac{3}{2}}$, respectively. 


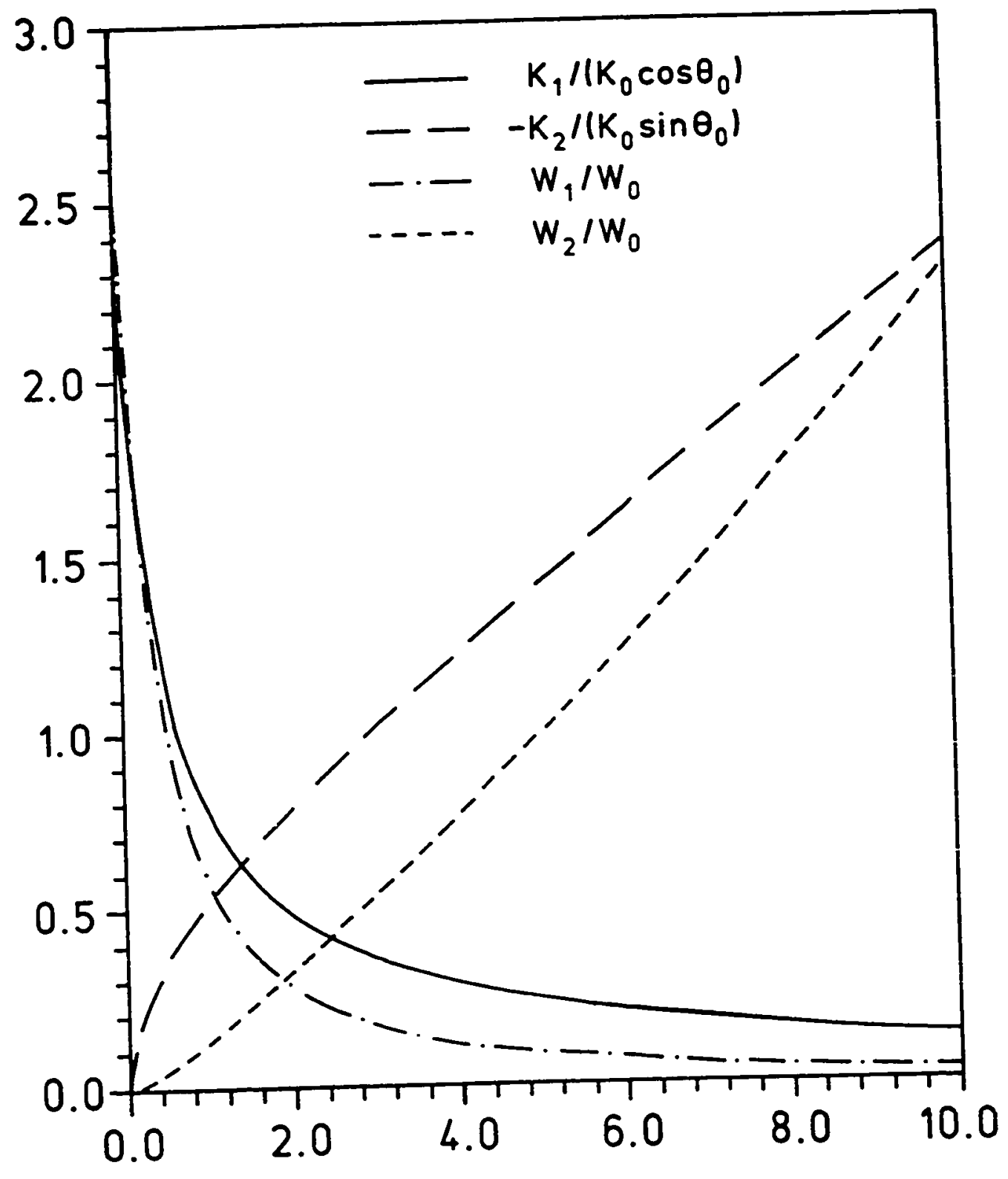

FIGURE 2. The variation of $K_{1} /\left(K_{0} \cos \theta_{0}\right)$.

$-K_{2} /\left(K_{0} \sin \theta_{0}\right), W_{1} / W_{0}$, and $W_{2} / W_{0}$ with the relative crack length $(C-B) / B$. 


\section{REFERENCES}

1. D.L. Bowie. Analysis of an infinite plate containing radial cracks at the boundary of an internal circular hole. J. Math. Phys. $\underline{35}(1956), 60-71$.

2. J. Tweed. Some dual equations with an application in the theory of elasticity. Jour. of Elasticity 2 (1972), 351-355.

3. D. Naylor. On Mellin type integral transforms. Jour. Math. and Mech. 12 (1963), 265-274.

4. J. Tweed and D.P. Rooke. The distribution of stress near a radial crack at the edge of a circular hole. Int. Jour. Engng. Sci. $\underline{11}$ (1973), 1185-1195.

5. J. Tweed and D.P. Rooke. The stress intensity factor for a crack at the edge of a loaded hole. Int. Jour. Solid Structures $\underline{15}$ (1979), 899-906.

6. I.N. Sneddon and M. Lowengrub. Crack Problems in the Classical Theory of Elasticity. (New York: John Wiley \& Sons, Inc., 1969).

7. J. Tweed. Thermoelasticity. (unpublished notes, Old Dominion University, Norfolk, Va., 1985).

8. H. Parkus. Thermoelasticity. (Blaisdell Publishing Company, Waltham, Mass., 1968.)

9. I.N. Sneddon. The Use of Integral Transforms. (McGraw-Hill Book Company, New York, N.Y., 1972.)

10. R.P. Srivastav and K.S. Parihar. Distribution of thermal stresses in wedge-shaped solids. Acta Mechanica 10 (1970), 145-160.

11. F. Erdogan and G.D. Gupta. On the numerical solution of singular integral equations. $\cong$. Appl. Math. 29 (1972), 525-534. 
12. A.L. Florence and J.N. Goodier. Thermal stresses due to uniform heat flow by an insulated ovaloid hole. J. Appl. Mech. 27 (1960), 635-639.

13. S.D. Conte and C. deBoor. Elementary Numerical Analysis.

(McGraw-Hill Book Company, New York, N.Y., 1980.)

14. C. deBoor. A Practical Guide to Splines. (Springer-Verlag, New York, N.Y., 1978.) 


\section{Appendix A}

\section{Sums of Series Involved in Calculating $f_{2}(r)$}

We wish to evaluate

$$
\begin{aligned}
S=\frac{3}{t r^{3}} & -\sum_{1}^{\infty} n(r t)^{-n-2}+t^{2} \sum_{n=2}^{\infty} n^{2}(r t)^{-n-2} \\
& -t^{4} \sum_{n=2}^{\infty} n(n+1)(r t)^{-n-2}+t^{2} \sum_{n=2}^{\infty} n^{2}(r t)^{-n} \\
& -\sum_{n=2}^{\infty} n(n-2)(r t)^{-n}
\end{aligned}
$$

Now

$$
\begin{aligned}
\sum_{n=1}^{\infty} n(r t)^{-n-2} & =\sum_{n=1}^{\infty} n z^{n+2}, \quad z=\frac{1}{r t} \\
& =z^{3} \sum_{n=0}^{\infty} n z^{n-1}=z^{3} \frac{d}{d z}\left(\sum_{0}^{\infty} z^{n}\right) \\
& =z^{3} \frac{d}{d z} \frac{1}{1-z}=\frac{z^{3}}{(1-z)^{2}} \\
& =\frac{z^{2}}{(z-1)^{2}}+\frac{z}{z-1}+z \\
& =\frac{1}{(1-r t)^{2}}+\frac{1}{1-r t}+\frac{1}{r t} .
\end{aligned}
$$

The following sums are obtained in a similar manner. 


$$
\begin{gathered}
\sum_{n=2}^{\infty} n^{2}(r t)^{-n-2}=\frac{-2}{(1-r t)^{3}}-\frac{1}{(1-r t)^{2}}-\frac{1}{1-r t} \\
-\frac{1}{r t}-\frac{1}{(r t)^{3}}, \\
\sum_{n=2}^{\infty} n(n+1)(r t)^{-n-2}=\frac{-2}{(1-r t)^{3}}-\frac{2}{(r t)^{3}}, \\
\sum_{n=2}^{\infty} n^{2}(r t)^{-n}=\frac{-1}{1-r t}+\frac{3}{(1-r t)^{2}}-\frac{2}{(1-r t)^{3}}-\frac{1}{r t}, \\
\sum_{n=2}^{\infty} n(n-2)(r t)^{-n}=\frac{-2}{(1-r t)^{2}}+\frac{1}{(1-r t)^{2}}+\frac{1}{1-r t}+\frac{1}{r t}
\end{gathered}
$$

Thus,

$$
S=\frac{\left(t^{2}-1\right)^{2}}{(1-r t)^{3}}+\frac{t^{2}-1}{(1-r t)^{2}}-\frac{t^{2}+1}{1-r t}-\frac{t^{2}+1}{r t}+\frac{t^{2}+1}{r^{3} t} .
$$

end 


\section{Appendix B}

\section{Some Useful Integrals}

The following integrals are readily obtainable by making the change of variable

$$
t=c^{-1} \cos ^{2} \theta+c \sin ^{2} \theta, \quad 0 \leq \theta \leq \frac{\pi}{2},
$$

where $c>0$ and $\Delta(t)=\sqrt{(c-t)\left(t-c^{-1}\right)}$.

$$
\begin{aligned}
& R_{1}(c)=\int_{1}^{c} \Delta(t) d t=\frac{1}{4}\left(\frac{c^{2}-1}{c}\right)^{2} \tan ^{-1} \sqrt{c}+\frac{1}{4}\left(c^{\frac{1}{2}}-c^{-\frac{1}{2}}\right)^{3} \\
& R_{2}(c)=\int_{1}^{c} t^{-1} \Delta(t) d t=\frac{(c+1)^{2}}{c} \tan ^{-1} \sqrt{c}-\left(c^{\frac{1}{2}}-c^{-\frac{1}{2}}\right)-\pi, \\
& R_{3}(c)=\int_{1}^{c} t^{-2} \Delta(t) d t=\frac{(c+1)^{2}}{c} \tan ^{-1} \frac{1}{\sqrt{c}}+\left(c^{\frac{1}{2}}-c^{-\frac{1}{2}}\right)-\pi . \\
& I_{1}(x)=\int_{1}^{c} \frac{d t}{\Delta(t)(t-x)} \\
& =2 \int_{\theta_{0}}^{\frac{\pi}{2}} \frac{\sec ^{2} \theta d \theta}{\left(c-c^{-1}\right) \tan ^{2} \theta+\left(c^{-1}-x\right) \sec ^{2} \theta} \\
& =2 \int_{\frac{1}{\sqrt{c}}}^{\infty} \frac{d u}{\left(c-c^{-1}\right) u^{2}-\left(x-c^{-1}\right)\left(1+u^{2}\right)} \\
& =\frac{2}{c-x} \int_{\frac{1}{\sqrt{c}}}^{\infty} \frac{d u}{u^{2}-\frac{x-c^{-1}}{c-x}}, \quad \theta_{0}=\sin ^{-1} \frac{1}{\sqrt{c+1}}, \quad c^{-1}<x<1 .
\end{aligned}
$$


Thus,

$$
\begin{aligned}
I_{1}(x)= & \int_{1}^{c} \frac{d t}{\Delta(t)(t-x)}=\frac{1}{\Delta(x)} \ln \left\{\frac{\sqrt{c-x}+\sqrt{c x-1}}{\sqrt{c-x}-\sqrt{c x-1}}\right\} \\
& c^{-1}<x<1
\end{aligned}
$$

Using (B.4) it is not difficult to show that for $c^{-1}<x<1$,

$$
\begin{aligned}
I_{2}(x)= & \int_{1}^{c} \frac{\Delta(t) d t}{t-x}=\Delta(x) \ln \left\{\frac{\sqrt{c-x}+\sqrt{c x-1}}{\sqrt{c-x}-\sqrt{c x-1}}\right\} \\
& -\frac{c-1}{\sqrt{c}}+\left(c+c^{-1}-2 x\right) \tan ^{-1} \sqrt{c} \\
I_{3}(x)= & \int_{1}^{c} \frac{\Delta(t) d t}{t(t-x)}=\frac{\Delta(x)}{x} \ln \left\{\frac{\sqrt{c-x}+\sqrt{c x-1}}{\sqrt{c-x}-\sqrt{c x-1}}\right\} \\
+ & \frac{2(x+1)}{x} \tan -1 \frac{1}{\sqrt{c}}-\pi, \\
I_{4}(x)= & \int_{1}^{c} \frac{\Delta(t) d t}{t^{2}(t-x)}=\frac{\Delta(x)}{x^{2}} \ln \left\{\frac{\sqrt{c-x}+\sqrt{c x-1}}{\sqrt{c-x}-\sqrt{c x-1}}\right\} \\
- & \frac{c-1}{\sqrt{c x}+\left\{\frac{2}{x^{2}}-\frac{c^{2}+1}{c x}\right\} \tan -\frac{1}{\sqrt{c}} \cdot}
\end{aligned}
$$

The following integrals are obtained by differentiating (B5)-(B7) with respect to $x$ : 


$$
\begin{aligned}
& J_{1}(x)=\int_{1}^{c} \frac{\Delta(t) d t}{(t-x)^{2}} \\
& =\frac{c+c^{-1}-2 x}{2 \Delta(x)} \ln \left\{\frac{\sqrt{c-x}+\sqrt{c x-1}}{\sqrt{c-x}-\sqrt{c x-1}}\right\} \\
& +\frac{c^{\frac{1}{2}}-c^{-\frac{1}{2}}}{1-x}-2 \tan ^{-1} \sqrt{c}, \quad c^{-1}<x<1 \text {, } \\
& J_{2}(x)=\int_{1}^{c} \frac{\Delta(t) d t}{t(t-x)^{2}} \\
& =\left\{\frac{c+c^{-1}-2 x}{2 x \Delta(x)}-\frac{\Delta(x)}{x^{2}}\right\} \ln \left\{\frac{\sqrt{c-x}+\sqrt{c x-1}}{\sqrt{c-x}-\sqrt{c x-1}}\right\} \\
& +\frac{c^{\frac{1}{2}}-c^{-\frac{1}{2}}}{x(1-x)}-\frac{2}{x^{2}} \tan ^{-1} \frac{1}{\sqrt{c}}, \quad c^{-1}<x<1, \\
& J_{3}(x)=\int_{1}^{c} \frac{\Delta(t) d t}{t^{2}(t-x)^{2}} \\
& =\left\{\frac{c+c^{-1}-2 x}{2 x^{2} \Delta(x)}-\frac{2 \Delta(x)}{x^{3}}\right\} \ln \left\{\frac{\sqrt{c-x}+\sqrt{c x-1}}{\sqrt{c-x}-\sqrt{c x-1}}\right\} \\
& +\frac{c^{\frac{1}{2}}-c^{-\frac{1}{2}}}{x^{2}(1-x)}+\frac{c-1}{\sqrt{c} x^{2}} \\
& +\left\{\frac{c^{2}+1}{c x^{2}}-\frac{4}{x^{3}}\right\} \tan ^{-1} \frac{1}{\sqrt{c}}, \quad c^{-1}<x<1 .
\end{aligned}
$$

By differentiating (B8) - (B10) with respect to $x$ we obtain:

$$
\begin{aligned}
K_{1}(x) & =\int_{1}^{=} \frac{\Delta(t) d t}{(t-x)^{3}}=\frac{\left(c^{\frac{1}{2}}-c^{-\frac{1}{2}}\right)^{3}(1+x)}{4(\Delta(x))^{2}(1-x)^{2}} \\
& -\frac{\left(c-c^{-1}\right)^{2}}{8(\Delta(x))^{3}} \ln \left\{\frac{\sqrt{c-x}+\sqrt{c x-1}}{\sqrt{c-x}-\sqrt{c x-1}}\right\}, \quad c^{-1}<x<1,
\end{aligned}
$$

54 


$$
\begin{aligned}
K_{2}(x) & =\int_{1}^{c} \frac{\Delta(t) d t}{t(t-x)^{3}} \\
& =\left\{\frac{\left(c+c^{-1}\right) x-2}{2 x^{3} \Delta(x)}-\frac{\left(c-c^{-1}\right)^{2}}{8 x(\Delta(x))^{3}}\right\} \ln \left\{\frac{\sqrt{c-x}+\sqrt{c x-1}}{\sqrt{c-x}-\sqrt{c x-1}}\right\} \\
& -\frac{\left(c^{\frac{1}{2}}-c^{-\frac{1}{2}}\right)(1-2 x)}{2 x^{2}(1-x)^{2}}+\frac{2}{x^{3}} \tan ^{-1} \frac{1}{\sqrt{c}} \\
& -\frac{\left\{\left(c+c^{-1}\right) x-2\right\}\left\{c^{\frac{1}{2}}-c^{-\frac{1}{2}}\right\}}{4 x^{2}(\Delta(x))^{2}(1-x)}, \quad c^{-1}<x<1,
\end{aligned}
$$

$$
\begin{aligned}
K_{3}(x)= & \int_{1}^{c} \frac{\Delta(t) d t}{t^{2}(t-x)^{3}} \\
= & \left\{\frac{3 \Delta(x)}{x^{4}}-\frac{\left(c-c^{-1}\right)^{2}}{8 x^{2}[\Delta(x)]^{3}}-\frac{c+c^{-1}-2 x}{x^{3} \Delta(x)}\right\} \\
& \cdot \ln \left\{\frac{\sqrt{c-x}+\sqrt{c x-1}}{\sqrt{c-x}-\sqrt{c x-1}}\right\}-\frac{c-1}{\sqrt{c} x^{3}} \\
+ & \frac{\left(c^{\frac{1}{2}}-c^{\left.-\frac{1}{2}\right)}\left(c+c^{-1}-2 x\right)\right.}{4 x^{2}(\Delta(x))^{2}(1-x)}-\frac{\left(c^{\frac{1}{2}}-c^{-\frac{1}{2}}\right)(4-5 x)}{2 x^{3}(1-x)^{2}} \\
+ & \left\{\frac{6}{x^{4}}-\frac{c^{2}+1}{c x^{3}}\right\} \tan ^{-1} \frac{1}{\sqrt{c}}, \quad c^{-1}<x<1 .
\end{aligned}
$$

In evaluating the following integrals we use the results in $(B 1)$ to $(B 13)$ 
The Integral $I I(r)$.

$$
\begin{aligned}
& I I(r)=\frac{1}{\pi} \int_{1}^{c} \Delta(t) \frac{1+t}{t^{2}}\left\{\frac{\left(t^{2}-1\right)^{2}}{(1-r t)^{3}}+\frac{t^{2}-1}{(1-r t)^{2}}-\frac{t^{2}+1}{1-r t}\right\} d t \\
&=\frac{1}{\pi} \int_{1}^{c} \Delta(t) \frac{(1+t)}{t^{2}} \frac{\left(t^{2}-1\right)^{2}}{(1-r t)^{3}} d t+\frac{1}{\pi} \int_{1}^{c} \Delta(t) \frac{(1+t)}{t^{2}} \frac{\left(t^{2}-1\right)}{(1-r t)^{2}} d t \\
&-\frac{1}{\pi} \int_{1}^{c} \Delta(t) \frac{(1+t)}{t^{2}} \frac{\left(t^{2}+1\right)}{(1-r t)} d t=I I_{3}(r)+I I_{2}(r)+I I_{1}(r) . \quad(\mathrm{B} 14) \\
& I I_{1}(r)=-\frac{1}{\pi} \int_{1}^{c} \frac{\Delta(t)}{1-r t}\left(t+1+\frac{1}{t}+\frac{1}{t^{2}}\right) d t \\
&=\frac{1}{\pi r} \int_{1}^{c} \frac{\Delta(t)}{t-r^{-1}}\left(\left[t-r^{-1}\right]+\left[1+r^{-1}\right]+\frac{1}{t}+\frac{1}{t^{2}}\right) d t . \quad(\mathrm{B} 15)
\end{aligned}
$$

Therefore,

$$
I I_{1}(r)=\frac{r^{-1}}{\pi}\left\{R_{1}(c)+\left(1+r^{-1}\right) I_{2}\left(r^{-1}\right)+I_{3}\left(r^{-1}\right)+I_{4}\left(r^{-1}\right)\right\}
$$

Similarly,

$$
\begin{aligned}
I I_{2}(r) & =\frac{r^{-2}}{\pi} \int_{1}^{c} \frac{\Delta(t)}{\left(t-r^{-1}\right)^{2}}\left\{t+1-\frac{1}{t}-\frac{1}{t^{2}}\right\} d t \\
& =\frac{r^{-2}}{\pi}\left\{I_{2}\left(r^{-1}\right)+\left(1+r^{-1}\right) J_{1}\left(r^{-1}\right)\right. \\
& \left.-J_{2}\left(r^{-1}\right)-J_{3}\left(r^{-1}\right)\right\}
\end{aligned}
$$

and 


$$
\begin{aligned}
I_{3}(r) & =\int_{1}^{c} \Delta(t)\left(\frac{1+t}{1-r t}\right)^{3}\left(1-\frac{1}{t}\right)^{2} d t \\
& =\frac{1}{\pi r^{3}} \int_{1}^{c} \Delta(t)\left\{\frac{1+r}{r-r t}-1\right\}^{3}\left(1-\frac{1}{t}\right)^{2} d t \\
& =\frac{1}{\pi} \int_{1}^{c} \Delta(t)\left\{\frac{-(1+r)^{3}}{r^{6}} \cdot \frac{1}{\left(t-r^{-1}\right)^{3}}-\frac{3(1+r)^{2}}{r^{5}} \cdot \frac{1}{\left(t-r^{-1}\right)^{2}}\right. \\
& \left.-\frac{3(1+r)}{r^{4}} \cdot \frac{1}{t-r^{-1}}-\frac{1}{r^{3}}\right\}\left(1-\frac{1}{t}\right)^{2} d t \\
& =\frac{-(1+r)^{3}}{\pi r^{6}}\left\{k_{1}\left(r^{-1}\right)-2 k_{2}\left(r^{-1}\right)+k_{3}\left(r^{-1}\right)\right\} \\
& -\frac{3(1+r)^{2}}{\pi r^{5}}\left\{J_{1}\left(r^{-1}\right)-2 J_{2}\left(r^{-1}\right)+J_{3}\left(r^{-1}\right)\right\} \\
& -\frac{3(1+r)}{\pi r^{4}}\left\{I_{2}\left(r^{-1}\right)-2 I_{3}\left(r^{-1}\right)+I_{4}\left(r^{-1}\right)\right\} \\
& -\frac{1}{\pi r^{3}}\left\{R_{1}(c)-2 R_{2}(c)+R_{3}(c)\right\}
\end{aligned}
$$




\section{The Integral III(t)}

$$
\begin{aligned}
I I I(t) & =\frac{1}{\pi} \int_{1}^{c} \Delta(\rho) \frac{1+\rho}{\rho^{2}}\left\{\frac{\left(\rho^{2}-1\right)^{2}}{2 \rho} \cdot \frac{1}{(1-\rho t)^{2}}+\frac{\rho^{2}-1}{\rho} \cdot \frac{1}{1-\rho t}\right. \\
& \left.+\frac{1-\rho^{2}}{2 \rho}+\frac{\rho^{2}+1}{\rho} \ln \left|\frac{\rho t-1}{\rho-1}\right|\right\} d \rho \\
& =\frac{1}{2 \pi} \int_{1}^{c} \Delta(\rho) \frac{\rho+1}{\rho}\left\{\left(t^{-2}-1\right)+\frac{2 t^{-1}\left(t^{-2}-1\right)}{\rho-t^{-1}}\right. \\
& \left.+\frac{\left(t^{-2}-1\right)^{2}}{\left(\rho-t^{-1}\right)^{2}}+2\left(1+\rho^{-2}\right) \ln \left|\frac{\rho t-1}{\rho-1}\right|\right\} d \rho \\
& =\frac{1}{2 \pi}\left(t^{-2}-1\right)\left[R_{1}(c)+R_{2}(c)\right]+\frac{1}{\pi} t^{-1}\left(t^{-2}-1\right)\left[I_{2}\left(t^{-1}\right)+I_{3}\left(t^{-1}\right)\right] \\
& +\frac{1}{2 \pi}\left(t^{-2}-1\right)^{2}\left[J_{1}\left(t^{-1}\right)+J_{2}\left(t^{-1}\right)\right] \\
& +\frac{1}{\pi} \int_{1}^{c} \Delta(\rho)\left(1+\rho^{-1}\right)\left(1+\rho^{-2}\right) \ln \left|\frac{\rho t-1}{\rho-1}\right| d \rho \\
& =\frac{1}{2 \pi}\left(t^{-2}-1\right)\left[R_{1}(c)+R_{2}(c)\right]+\frac{1}{\pi} t^{-1}\left(t^{-2}-1\right)\left[I_{2}\left(t^{-1}\right)+I_{3}\left(t^{-1}\right)\right] \\
& +\frac{1}{2 \pi}\left(t^{-2}-1\right)^{2}\left[J_{1}\left(t^{-1}\right)+J_{2}\left(t^{-1}\right)\right]+I I I_{1}(t) ;
\end{aligned}
$$




$$
\begin{aligned}
I I I_{1}(t) & =\frac{1}{\pi} \int_{1}^{c} \Delta(\rho)\left(1+\rho^{-1}\right)\left(1+\rho^{-2}\right) \ln \left|\frac{\rho t-1}{\rho-1}\right| d \rho \\
& =\frac{4\left(c^{\frac{1}{2}}-c^{-\frac{1}{2}}\right)}{\pi(c-1)} \int_{1}^{c} \ln \left|\frac{\rho t-1}{\rho-1}\right|(c-\rho) d \rho \\
& +\frac{1}{\pi} \int_{1}^{c}\left\{\Delta(\rho)\left(1+\rho^{-1}\right)\left(1+\rho^{-2}\right)\right. \\
& \left.-4\left(c^{\frac{1}{2}}-c^{-\frac{1}{2}}\right) \frac{c-\rho}{c-1}\right\} \ln \left|\frac{\rho t-1}{\rho-1}\right| d \rho \\
& =\frac{4}{\pi \cdot \sqrt{c}} \int_{1}^{c} \ln \left|\frac{\rho t-1}{\rho-1}\right|(c-\rho) d \rho \\
& +\frac{1}{\pi} \int_{1}^{c}\left\{\Delta(\rho)\left(1+\rho^{-1}\right)\left(1+\rho^{-2}\right)\right. \\
& \left.-\frac{4}{\sqrt{c}}(c-\rho)\right\} \ln \left|\frac{\rho t-1}{\rho-1}\right| d \rho .
\end{aligned}
$$

Hence, after a little manipulation, we find that

$$
\begin{aligned}
I I I(t) & =\frac{1}{2 \pi}\left(t^{-2}-1\right)\left[R_{1}(c)+R_{2}(c)\right]+\frac{1}{\pi} t^{-1}\left(t^{-2}-1\right)\left[I_{2}\left(t^{-1}\right)+I_{3}\left(t^{-1}\right)\right] \\
& +\frac{1}{2 \pi}\left(t^{-2}-1\right)^{2}\left[J_{1}\left(t^{-1}\right)+J_{2}\left(t^{-1}\right)\right] \\
& +\frac{2}{\pi \sqrt{c}}\left\{(c-1)^{2} \ln \left|\frac{t-1}{c-1}\right|+\left(c-t^{-1}\right)^{2} \ln \left|\frac{c t-1}{t-1}\right|\right. \\
& \left.-(c-1)\left(1-t^{-1}\right)\right\}+\frac{1}{\pi} \int_{1}^{c}\left\{\Delta(\rho)\left(1+\rho^{-1}\right)\left(1+\rho^{-2}\right)\right. \\
& \left.-\frac{4}{\sqrt{c}}(c-\rho)\right\} \ln \left|\frac{\rho t-1}{\rho-1}\right| d \rho
\end{aligned}
$$

where the integral in (B21) may be approximated to a high degree of accuracy using cubic splines. 


\title{
Appendix C
}

\author{
PROCRAM SIRESS \\ TMPLICIT REAL*8 $(\mathrm{A}-\mathrm{G})$, REPL $* 8(\mathrm{O}-\mathrm{Z})$ \\ TMPLICIT INIEGER*4 (H-N)
}
C Given the length of the crack, $\mathrm{Cl}$, and the desired C number of Gaussian points, N, STREss constructs vectors C containing the abscissas of the Gaussian points $T(j)$ and C the collocation points $Y(j)$. STrEss then calls subroutine C SYS1 or SYS2 which returns the opening mode stress C intensity and crack energy or the sliding mode intensity C and crack energy, respectively. These are stored in data C files which are used to construct graphs of the stress C intensities and crack energies for various crack lengths C using GRAPHER, a computer graphing package by Golden C Graphics Software, Inc.

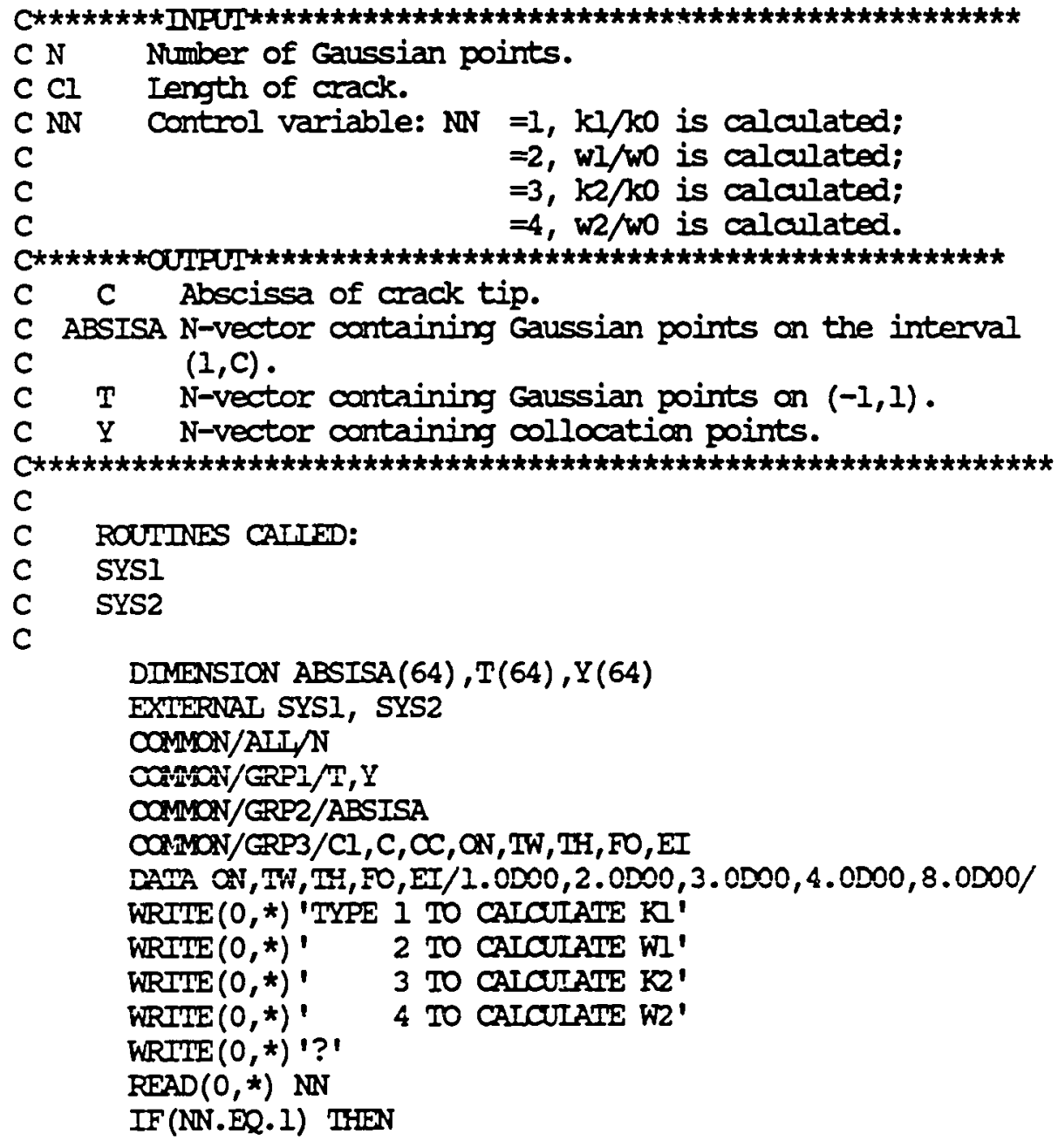




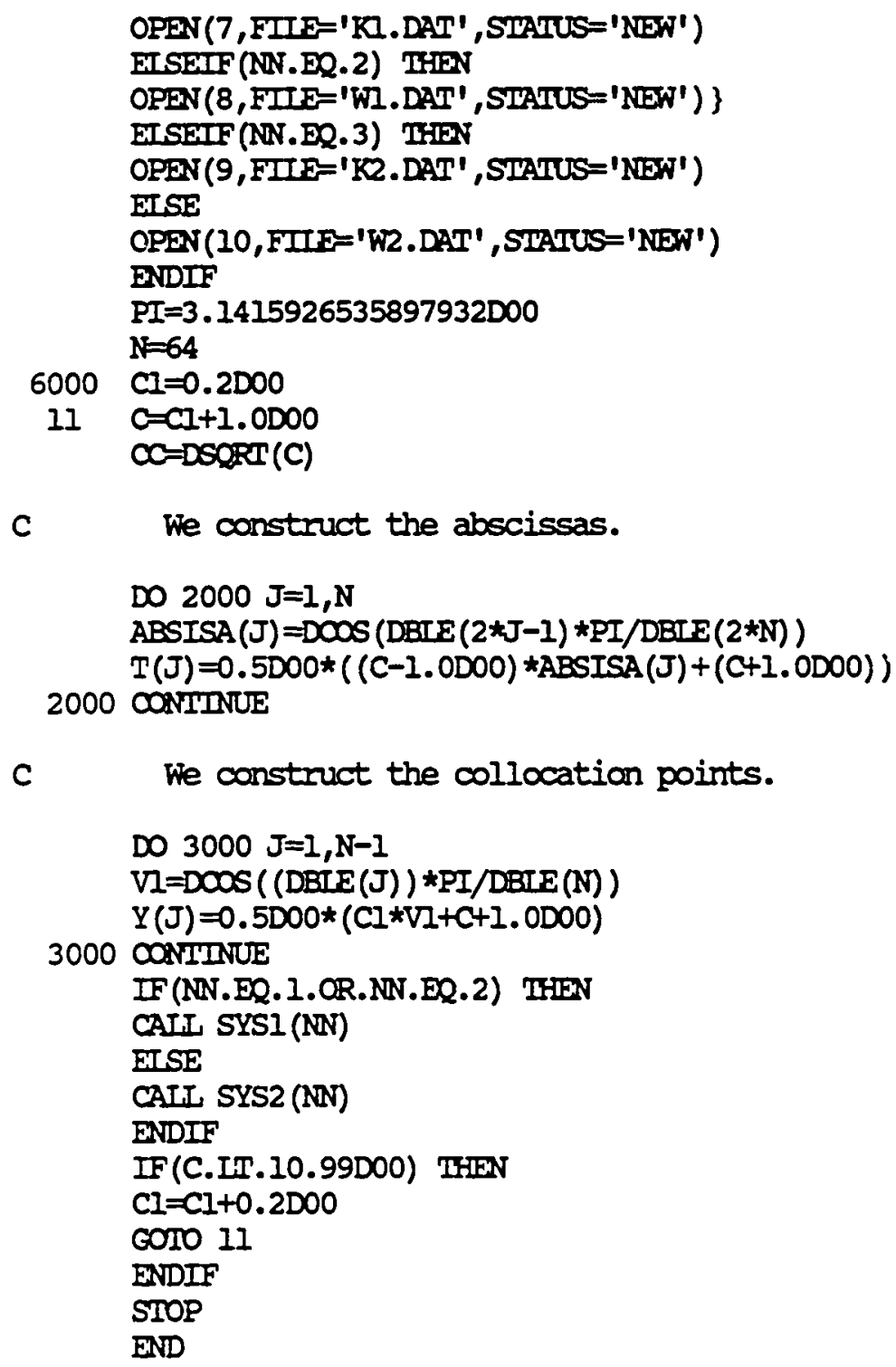


SUBROUIINE SYSI (NRI)

IMPLICIT REAI*8 (A-G), REAL $* 8(O-Z)$

IMPLICIT INIESEER*4 ( $\mathrm{H}-\mathrm{N})$

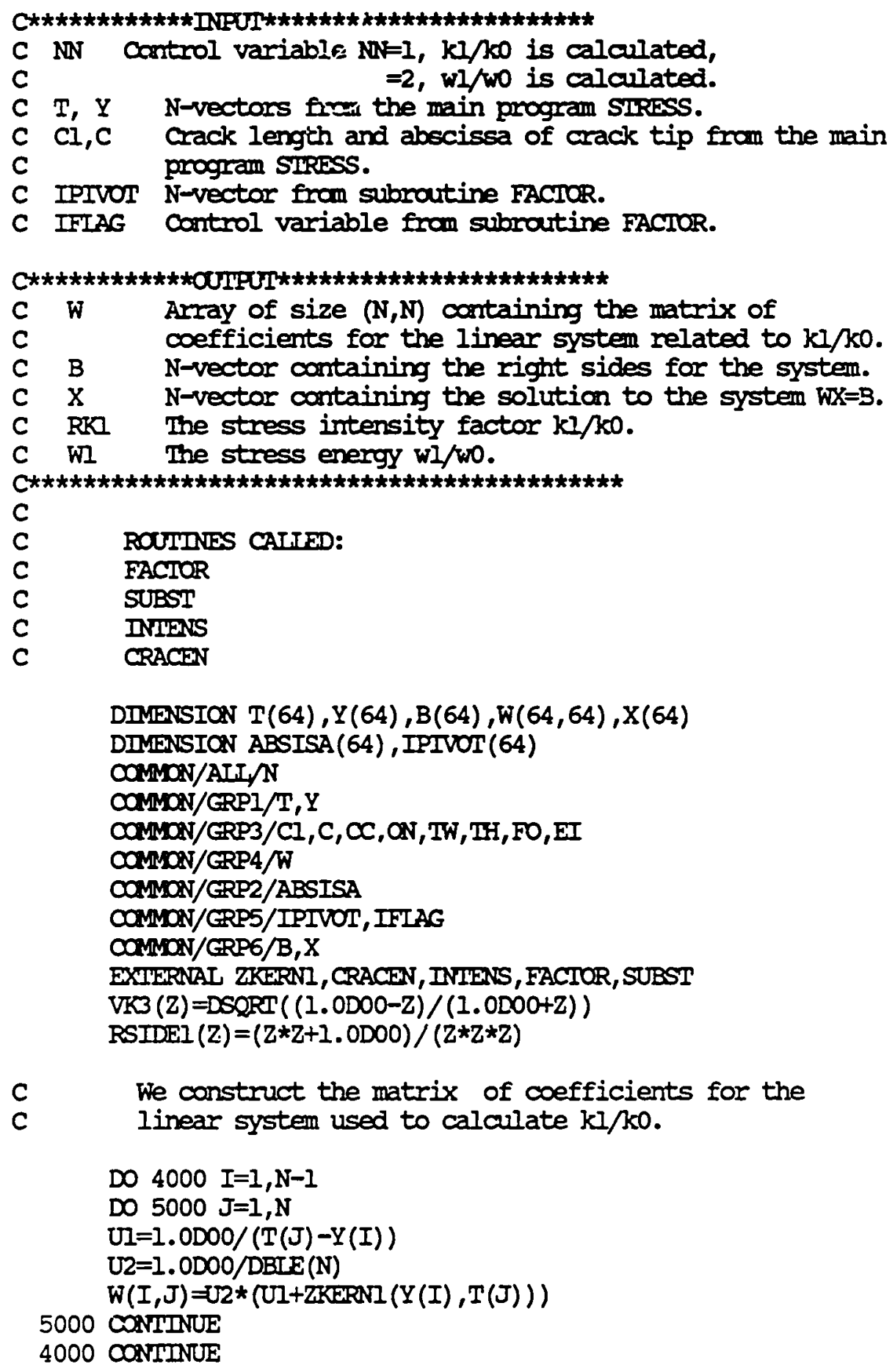




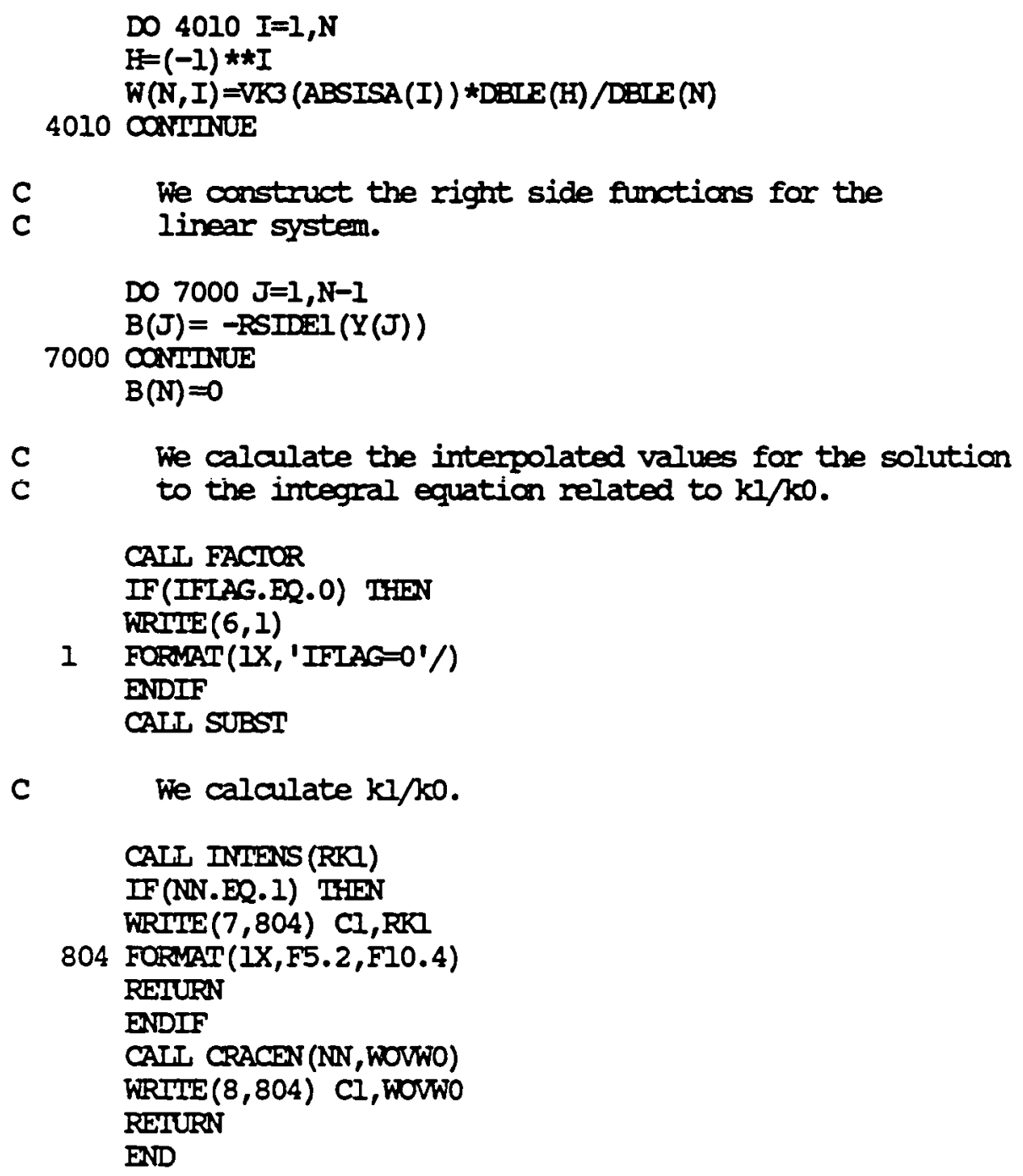




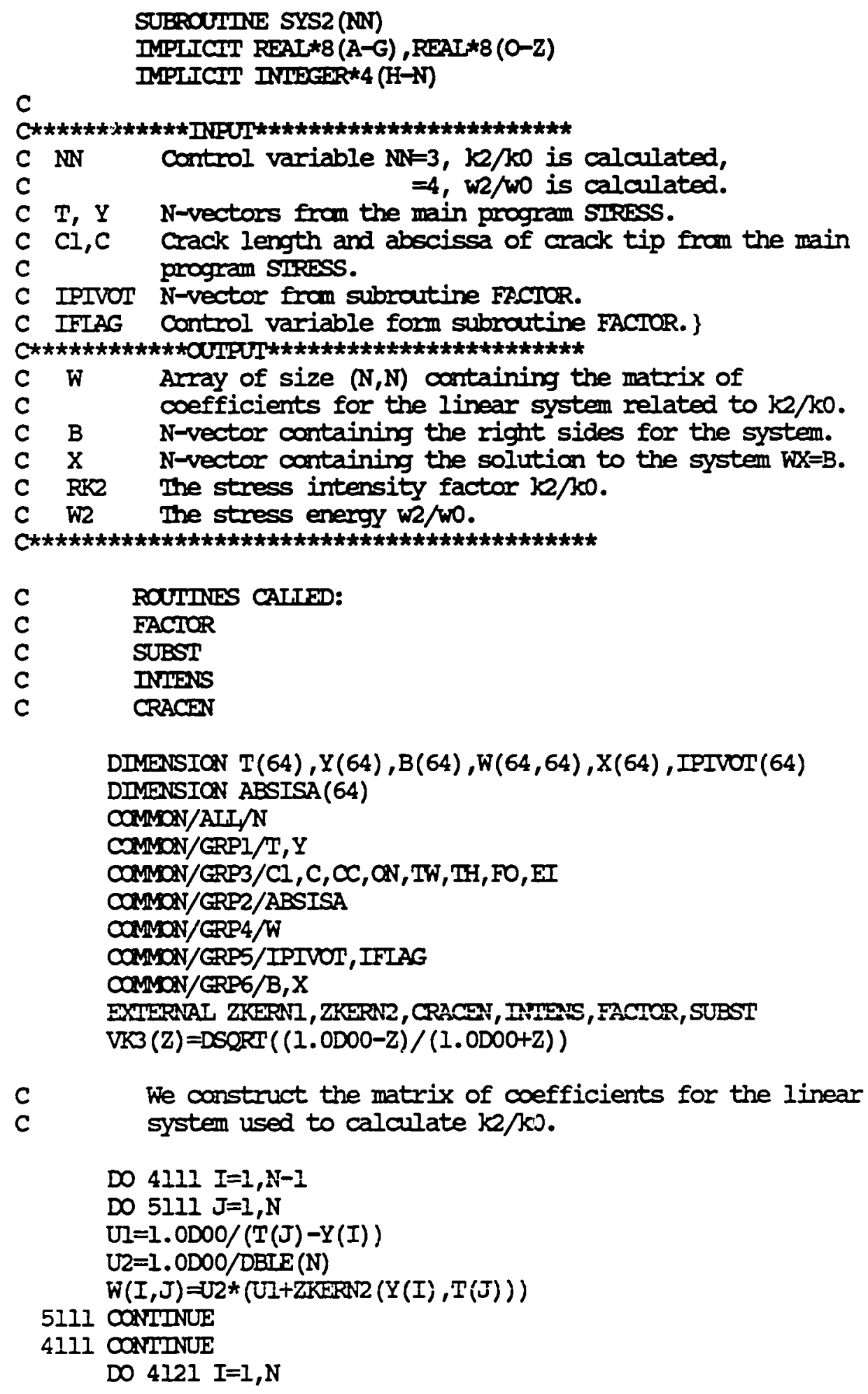

C We construct the matrix of coefficients for the linear

C system used to calculate $12 / \mathrm{kJ}$. 


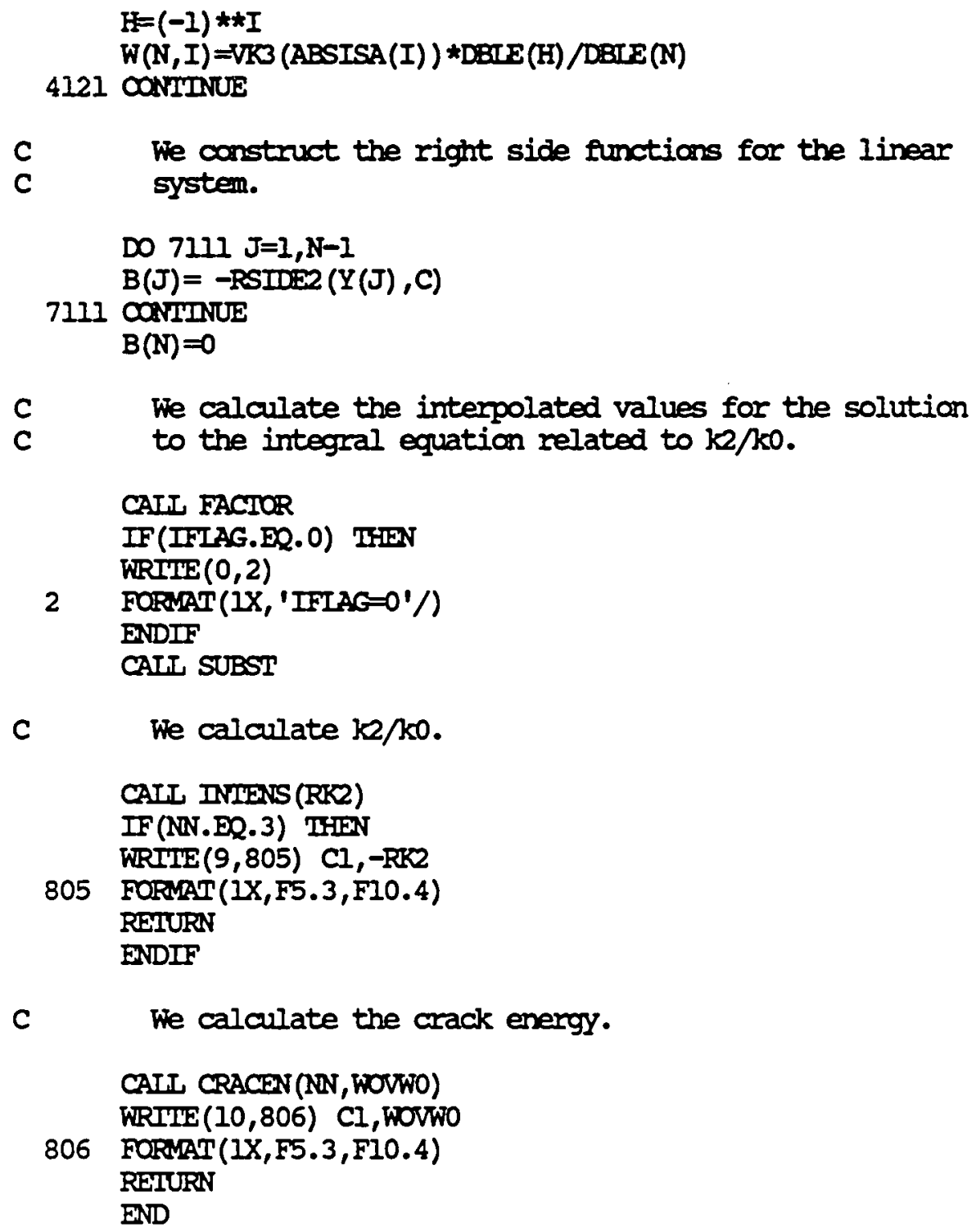




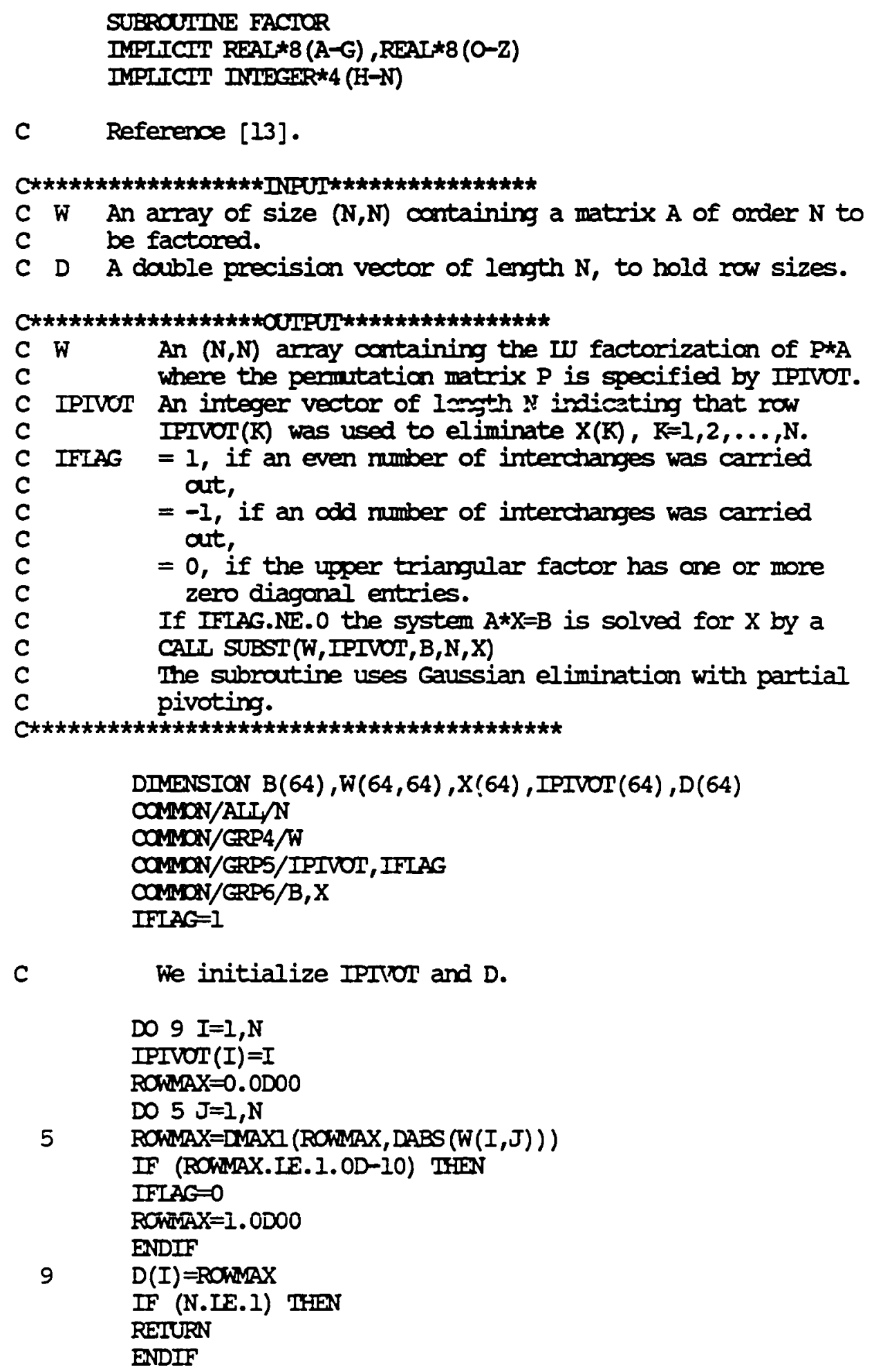


DO $20 \quad R=1, N-1$

C We determine the pivot row-the row ISTAR.

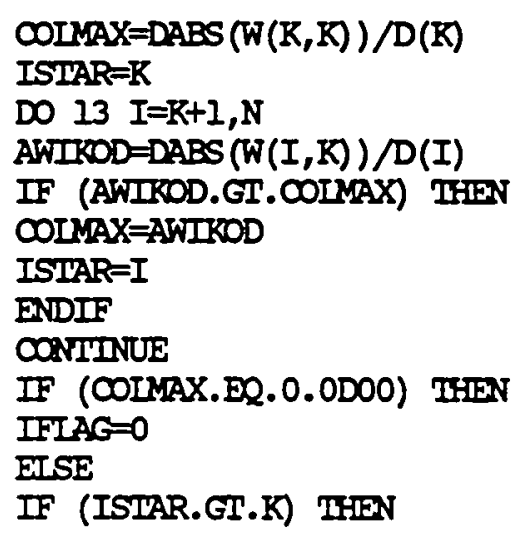

C We make $\mathrm{K}$ the pivot row by interchanging it with

$\mathrm{C}$ the chosen row ISTAR.

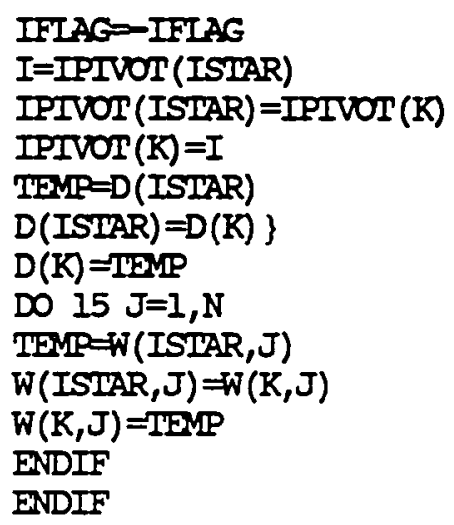

C

$$
\text { We eliminate } X(K) \text { from rows } K+1, \ldots, N \text {. }
$$

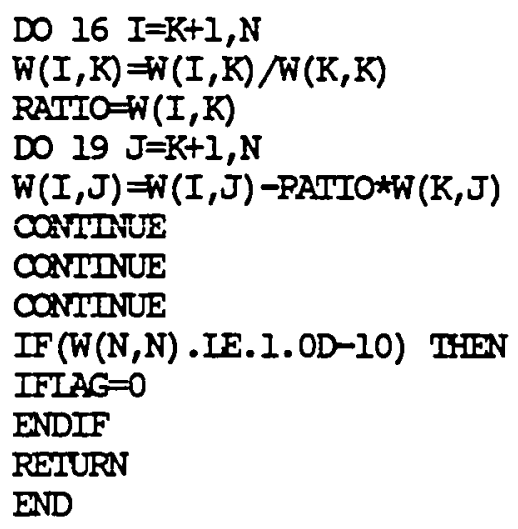




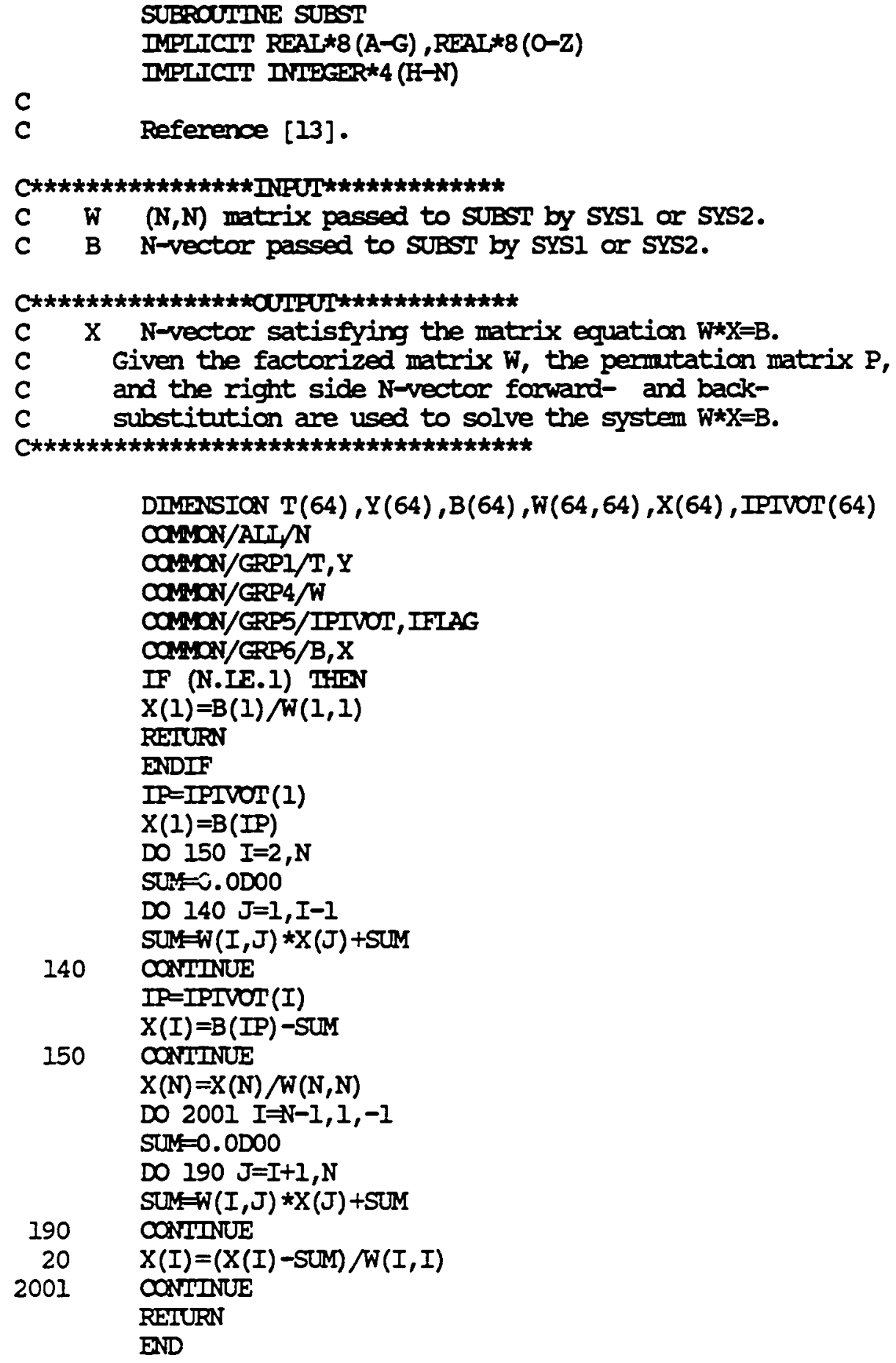


SUBROUTINE CRACEN (NN, WOWWO)

TMPIICIT REAI $\star 8(A-G), R E A L \star 8(O-Z)$

IMPLICTT INIDEER $* 4(\mathrm{H}-\mathrm{N})$

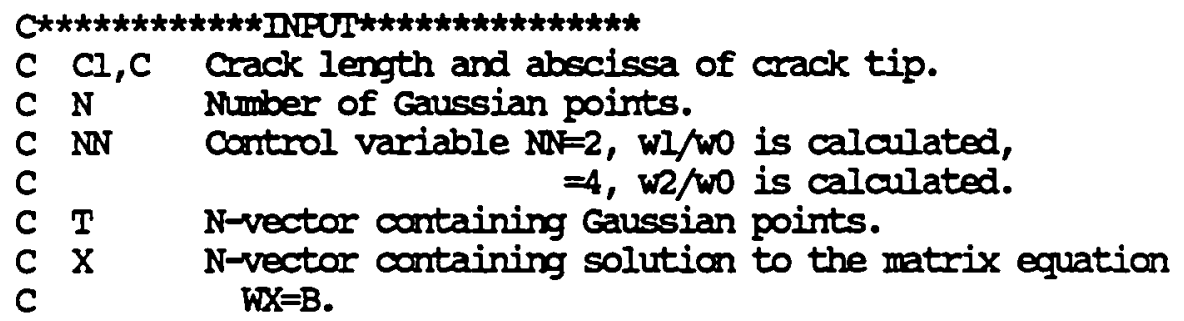

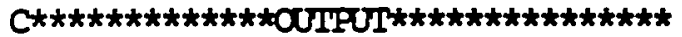

$C$ WOWWO w1/WO if $N=2, W 2 / W 0$ if $N N=4$.

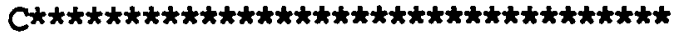

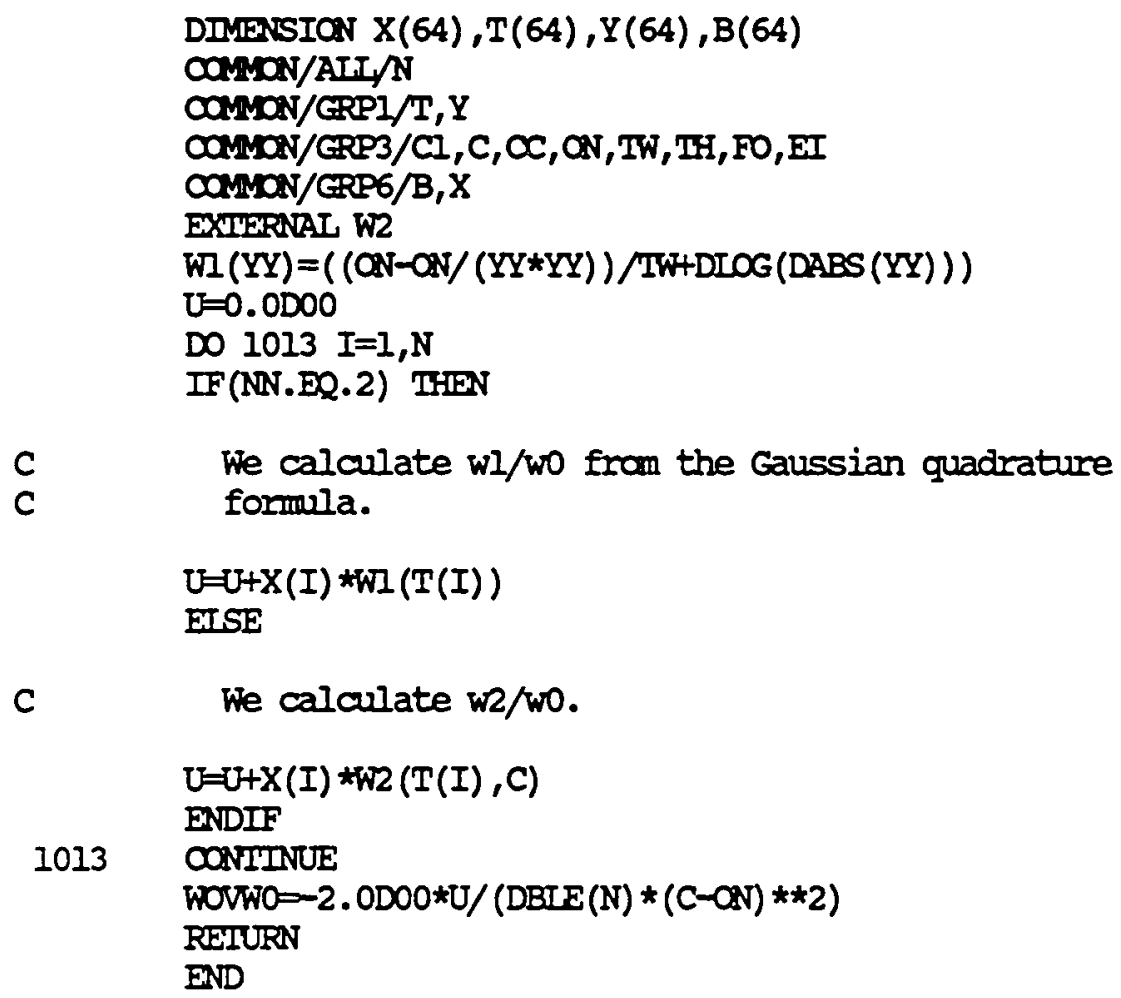


SUBROUTINE INTIENS(RK)

TMPIICIT REAL $* 8(A-G), R E A L * 8(O-Z)$

IMPLICIT INTECER $* 4(\mathrm{H}-\mathrm{H})$

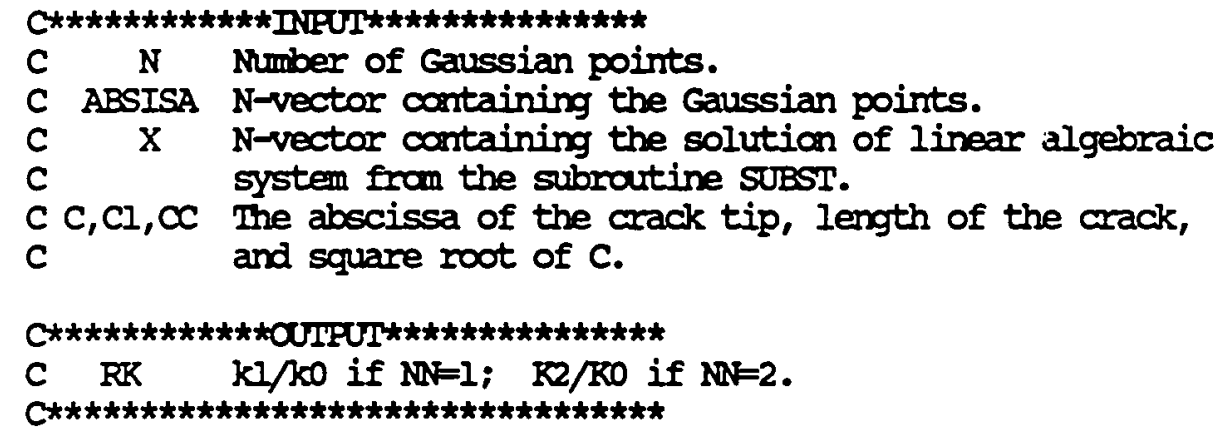

C We calculate the stress intensity factor from the c Chebyshev-Iagrange interpolation formula.

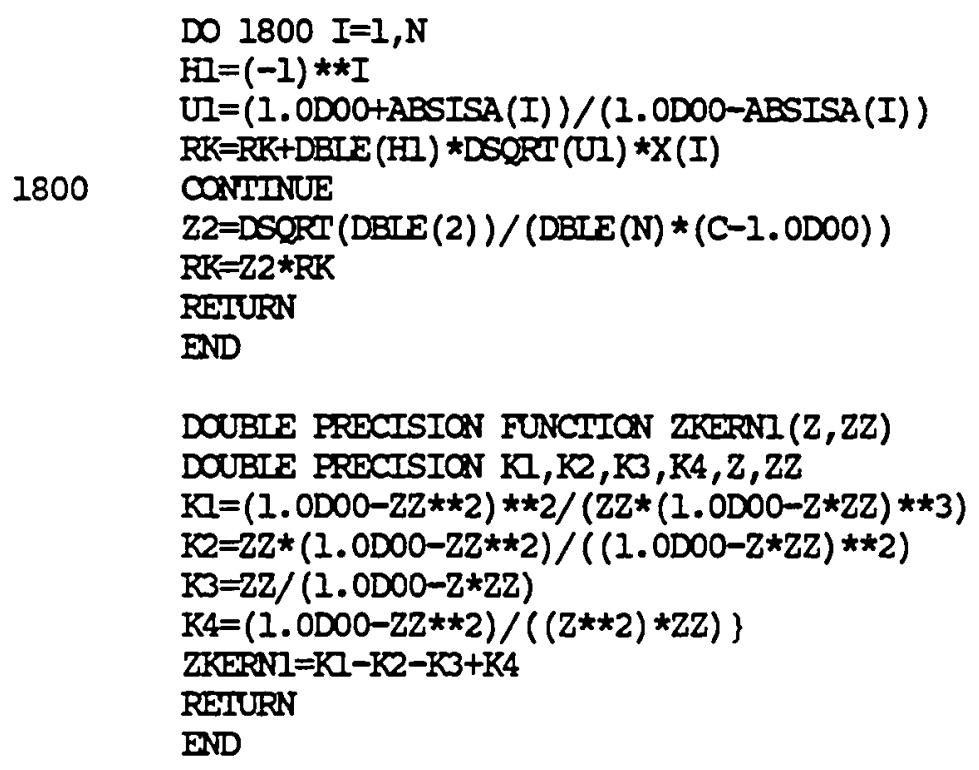


DOUBLE PRECISION FUNCTION ZKKERN2 $(\mathrm{Z}, \mathrm{ZZ})$

DOUBIE PRECISION $\mathrm{K} L, \mathrm{~K} 2, \mathrm{~K} 3, \mathrm{Z}, \mathrm{ZZ}, \mathrm{U}$

$\mathrm{K}=(1.0 \mathrm{DOO}-\mathrm{ZZ} * \star 2) * 2 /(\mathrm{ZZ} *(1.0 \mathrm{DOO}-\mathrm{Z} * \mathrm{ZZ}) \star \star 3)$

$\mathrm{K} 2=(1.0 \mathrm{DOO}-\mathrm{ZZ} * * 2) /(\mathrm{ZZ} *(1.0 \mathrm{DOO}-\mathrm{Z} * \mathrm{ZZ}) * * 2)$

$\mathrm{K} 3=\mathrm{ZZ} /(1.0 \mathrm{DOO}-\mathrm{Z} * \mathrm{ZZ})$

ZKEERNZ=KZ-K2-K3

REIURN

END

SUBROUIINE CUBSPL(T, ENDPT,Q)

IMPLICIT REAL*8 (A-G), REAI*8(O-Z)

INPICIT INIEGER*4(H-N)

C

C Reference [14].

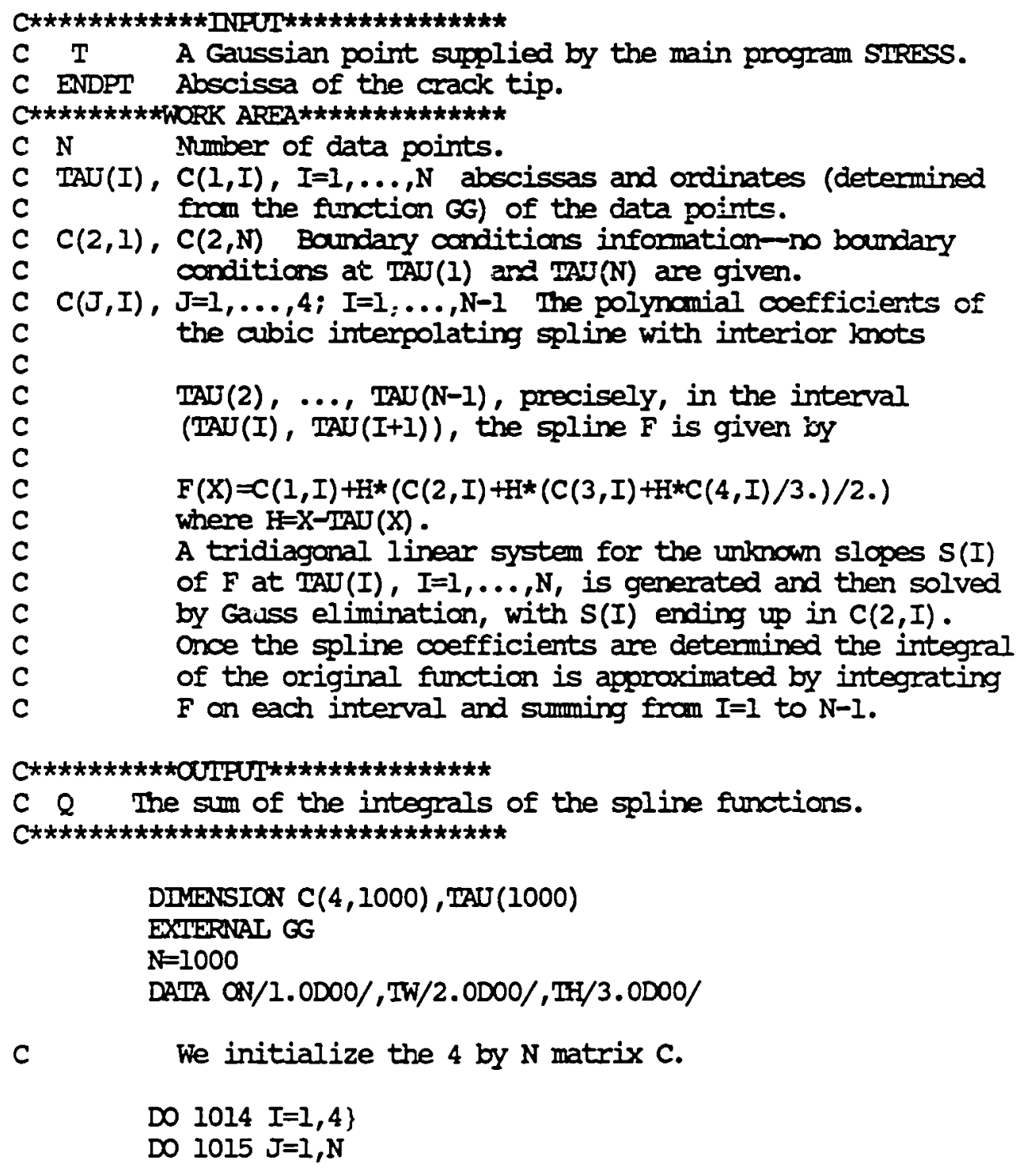




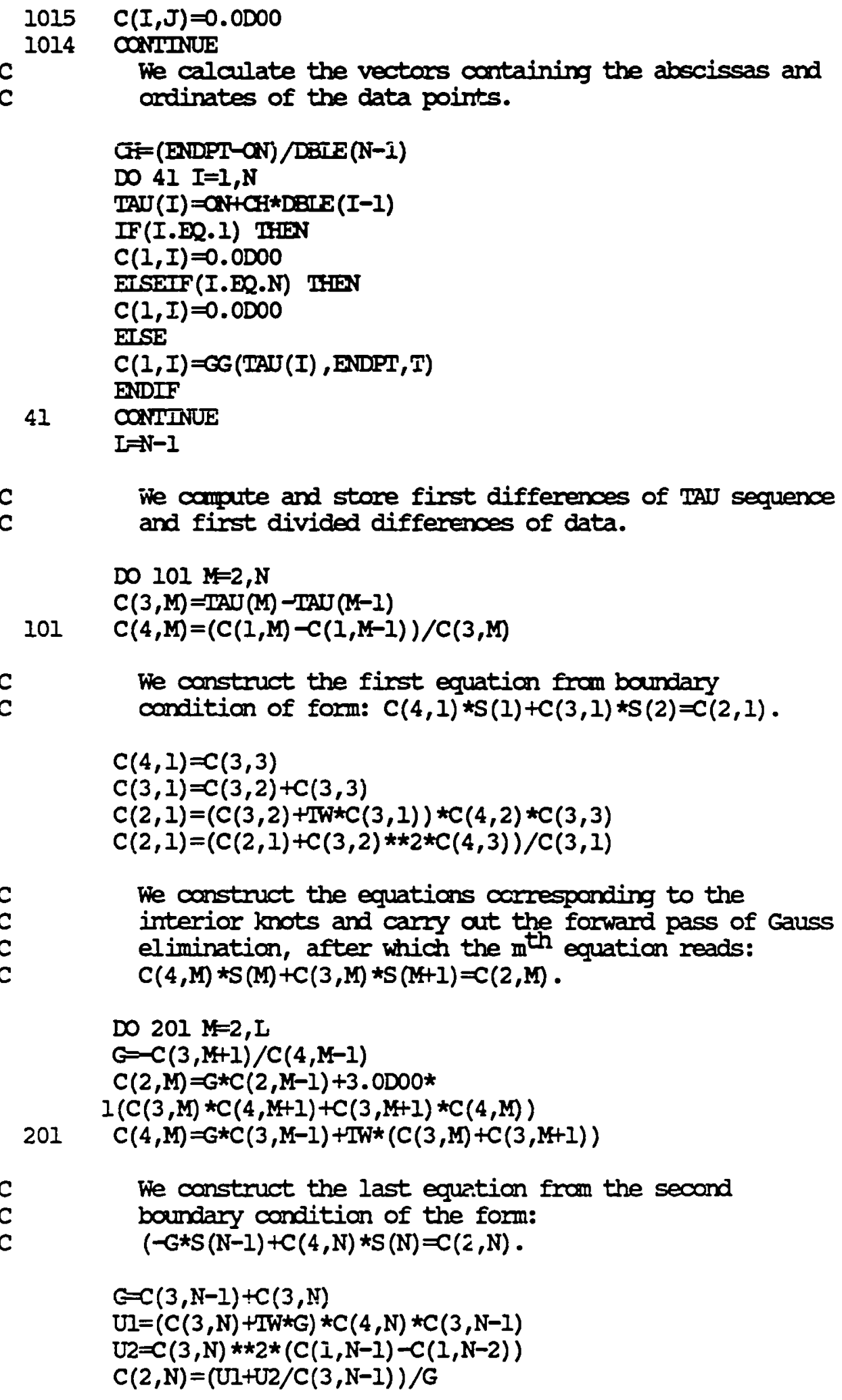




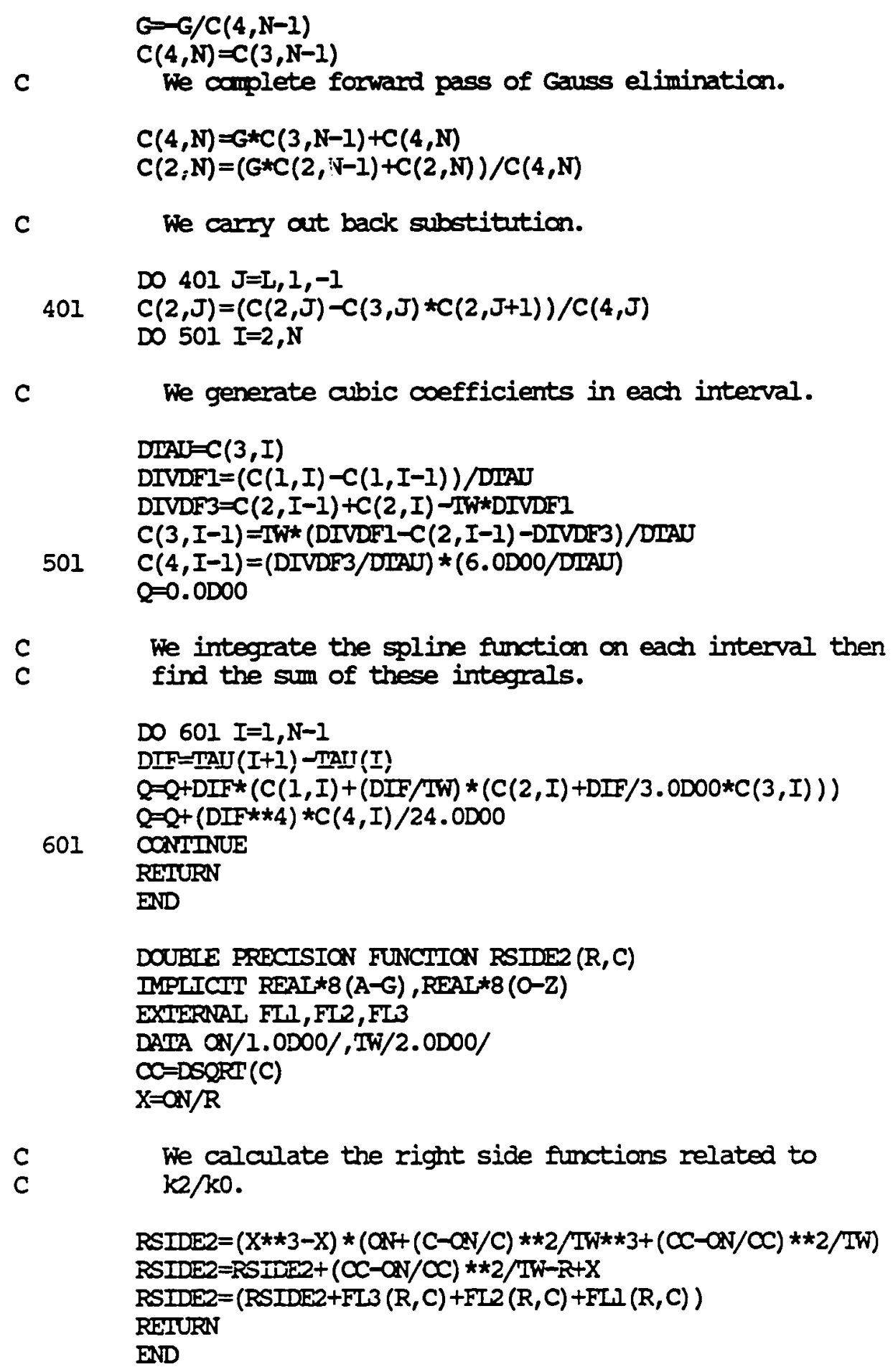




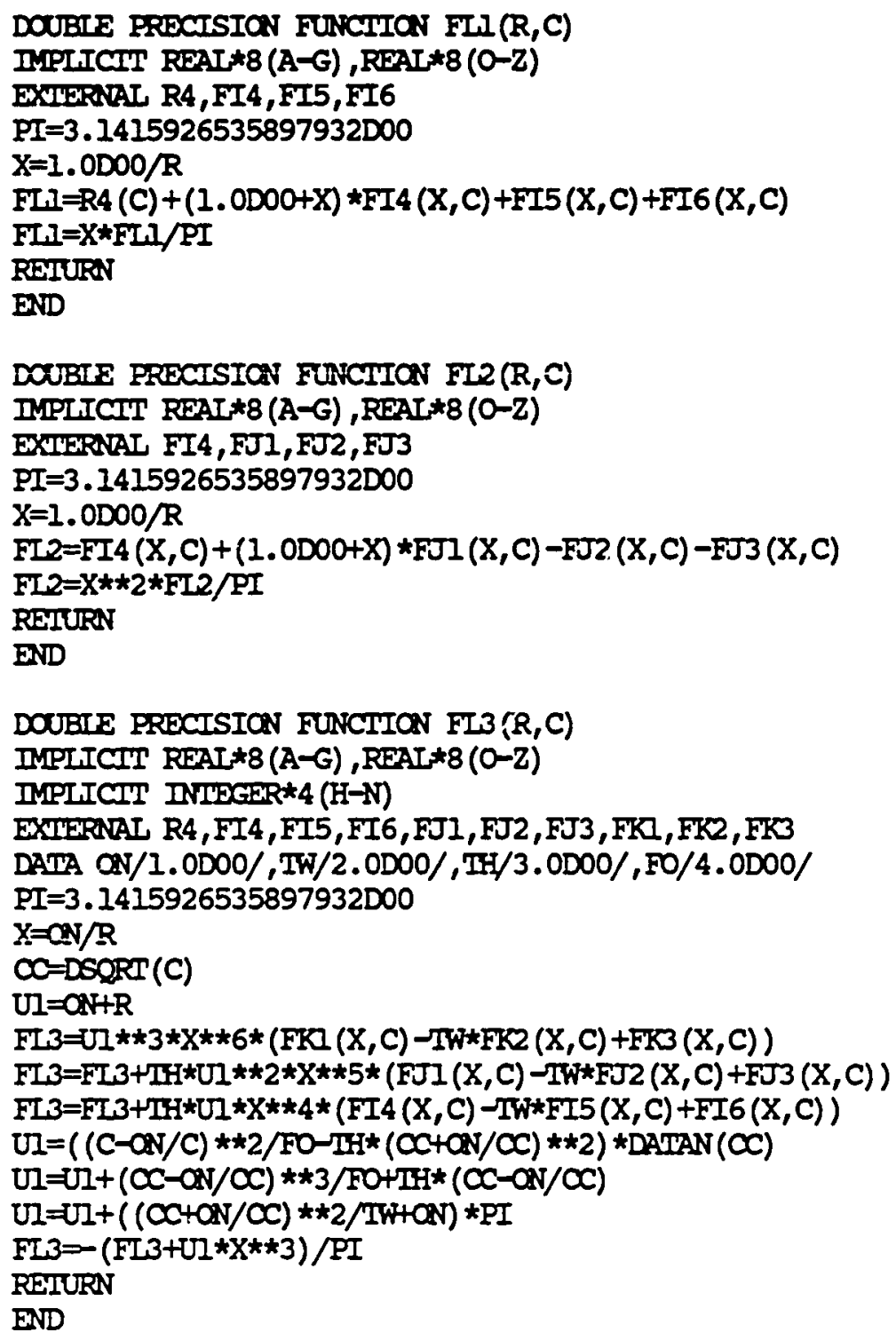




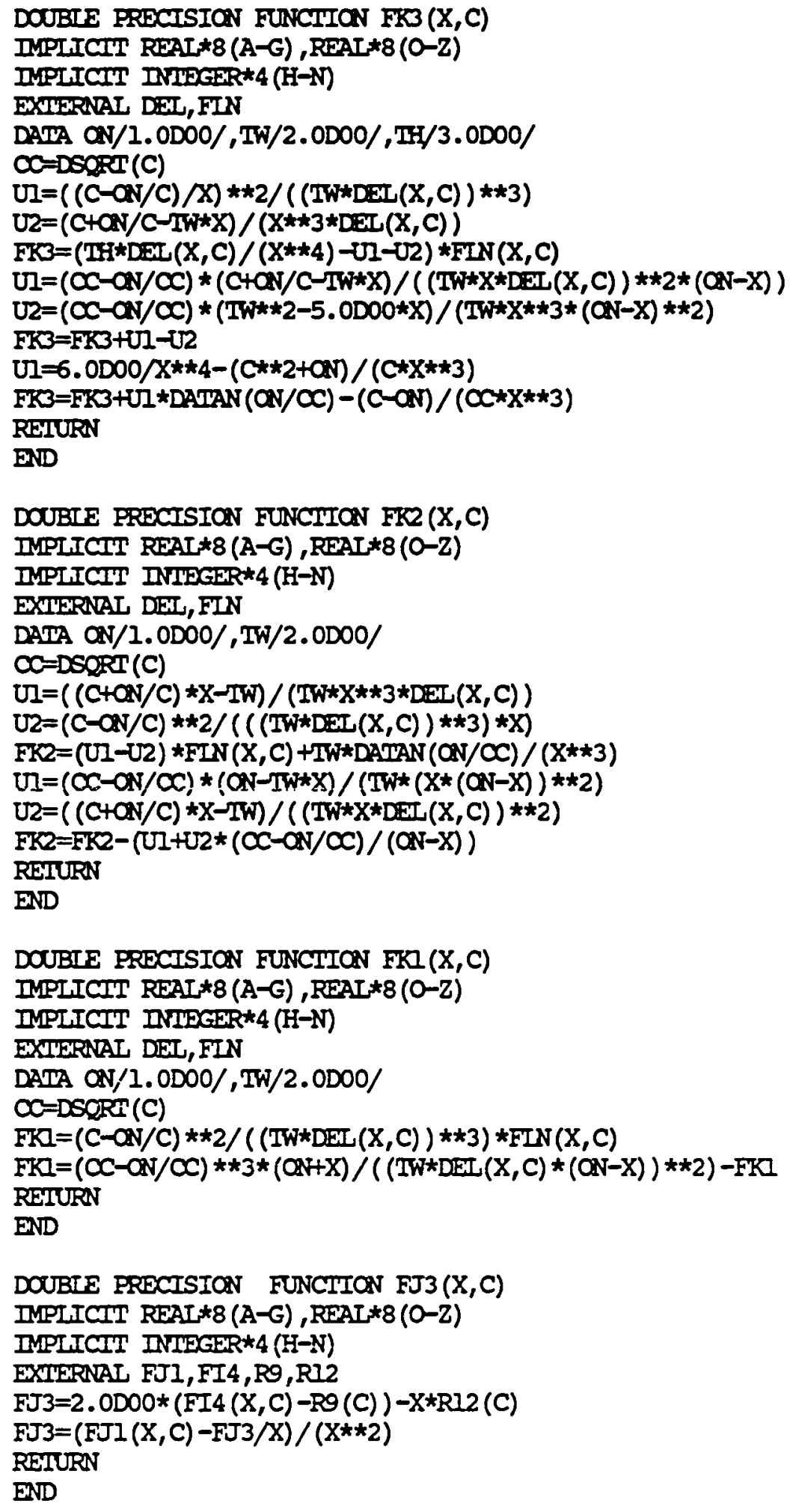




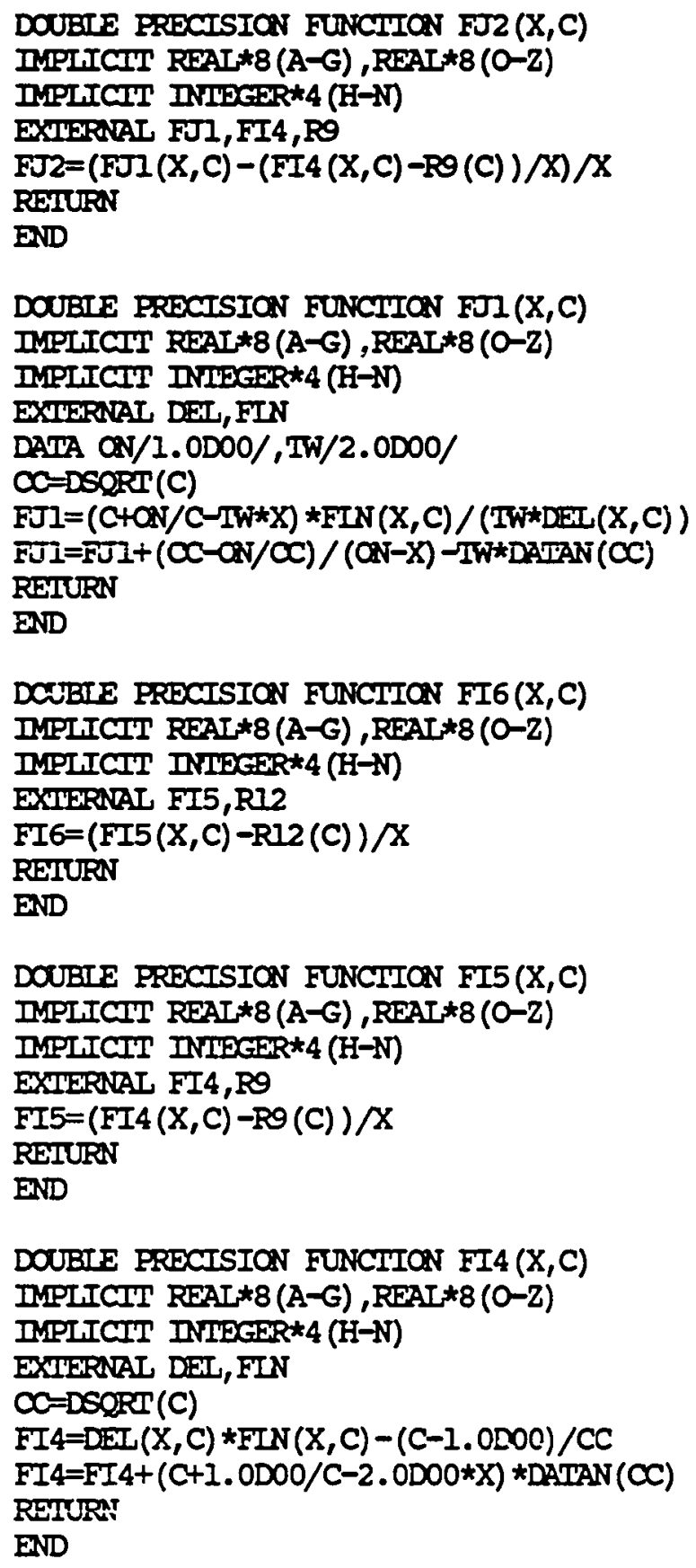




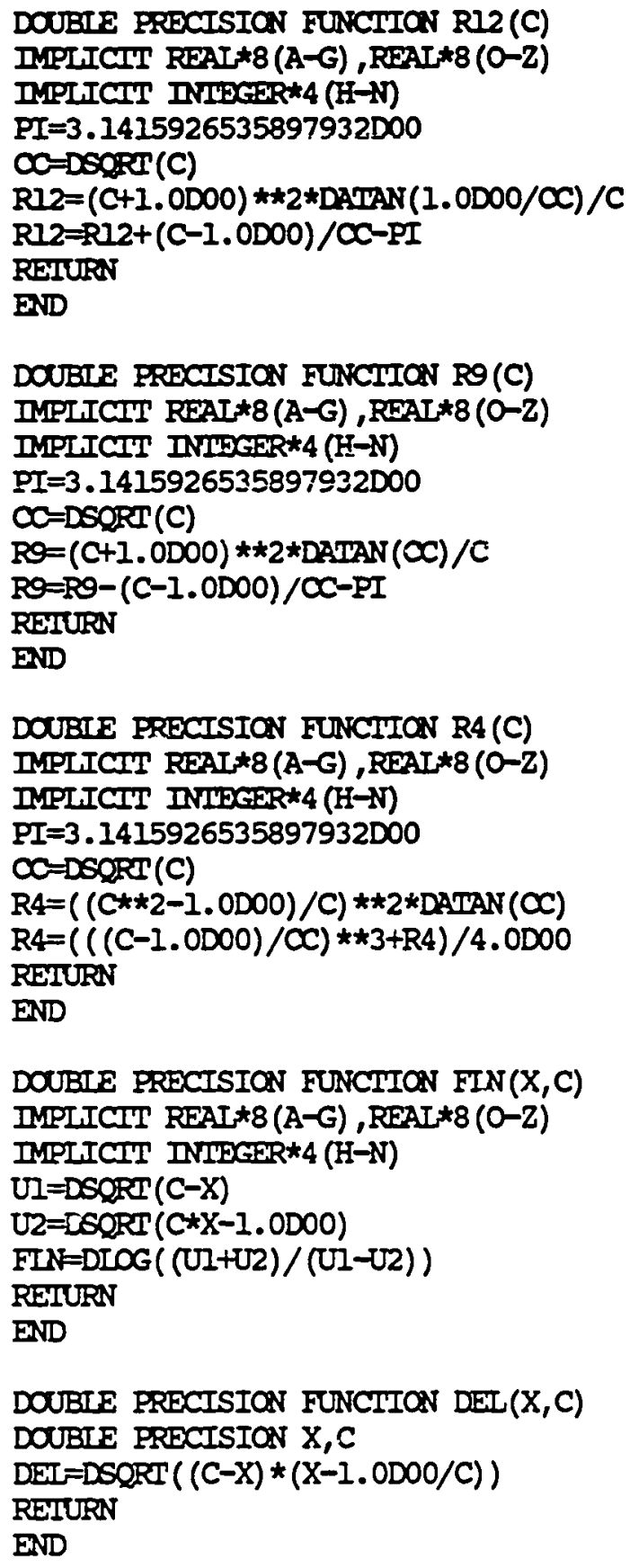




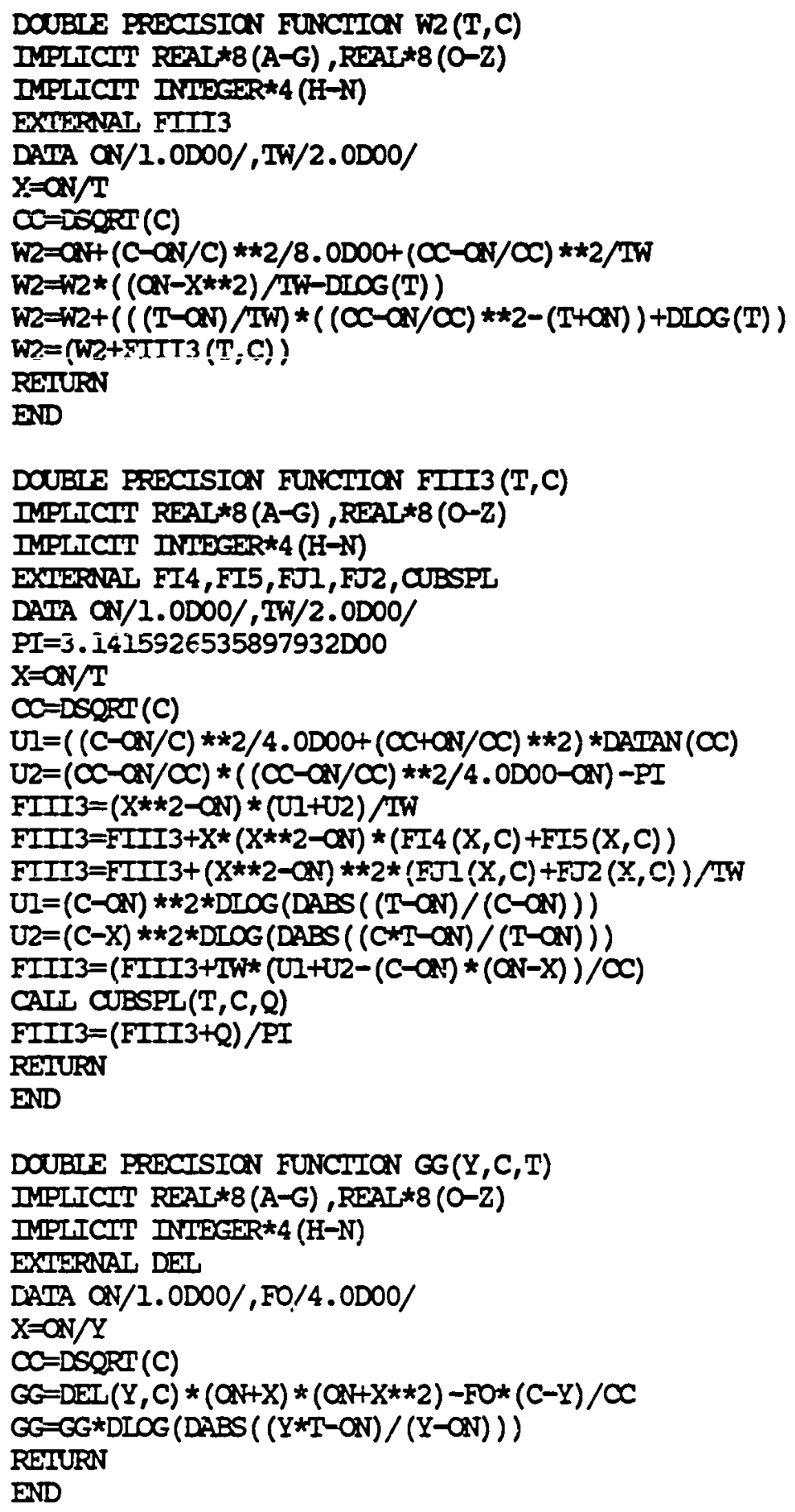




\section{Autobiographical Statement}

The author was born in Tillery, North Carolina on May 10, 1928. He received the Bachelor of Science degree from Hampton Institute, Hampton, Virginia in social science with a minor in mathematics in 1955, a Master of Science degree in mathematics from New York University, New York, New York in 1957, and a Certificate of Advanced Graduate Studies in mathematics and science from w̌esieyan üniversity, Middletown, Connecticut in 1965. The author has served as Supervisor of Mathematics Instruction for the public schools of Glastonbury, Connecticut and of Hingham, Massachusetts. He has also served as Chairman of the Mathematics Section of the Curriculum Development and Research Center, Kenya Ministry of Education, Nairobi, Kenya. He is the author of articles in The Arithmetic Teacher and The Mathematics Teacher, journals of the National Council of Teachers of Mathematics, Co-author of Patterns in Mathematics, Houghton Mifflin, Boston, Massachusetts, 1965, and co-author of Harper \& Row Mathematics, Harper \& Row, Publishers, New York, N.Y., 1982. 